\title{
Transition Probabilities and Oscillator Strengths for E1 Transitions of Ce III
}

\author{
B. KaraÇOBAN Usta* \\ Department of Physics, Sakarya University, 54187, Sakarya, Turkey \\ (Received August 29, 2014; in final form February 13, 2015)
}

\begin{abstract}
We report calculations of wavelengths, oscillator strengths, and transition probabilities (or rates) for electric dipole (E1) transitions in doubly ionized cerium (Ce III, $Z=58$ ), using the relativistic Hartree-Fock method developed by Cowan. Comparisons are made with the previously reported available calculations and experiments in literature.
\end{abstract}

DOI: $10.12693 /$ APhysPolA.127.1605

PACS: 31.15.ag, 31.15.aj, 31.30.-i, 32.70.Cs

\section{Introduction}

The lanthanide elements $(Z=57-71)$ are important in astrophysics in relation to nucleosynthesis and star formation considerations. Spectroscopic investigations of the doubly ionized elements of this group are motivated by astrophysical interest because, in the hot chemically peculiar (CP) stars, there is an overwhelming presence of lines belonging to the third spectrum which corresponds to the dominant ionization stage [1].

The rare-earth element cerium has a particularly rich, even by rare-earth standards, optical emission spectrum from both its neutral and singly ionized stages. This characteristic has made cerium attractive for use in highintensity discharge light sources where it improves both luminous efficacy and colour rendering [2].

The works for energy levels and lifetimes of doubly ionized cerium (Ce III) can be found in, for example, [3-7]. Many strong lines of cerium in the ultraviolet evidently arise from doubly ionized atoms. King and King published a list of these [8]. Triplet and singlet terms of Ce III were reported by Russell et al. [9]. Observations of the third spectrum of cerium from 757 to $11091 \AA$ were given by Sugar [10]. The $4 f^{2}-4 f 5 d$ lines of Ce III were measured by Johansson and Litzén [11]. Bord and co-workers calculated oscillator strengths for some Ce III lines [12]. Later, the spectroscopic analysis of Ce III was extended. Wyart and Palmeri gave a list of computed oscillator strengths for some selected transitions [6]. Transition probabilities for Ce III were obtained from branching fractions calculated by Cowan code and experimental lifetimes by $\mathrm{Li}$ and co-workers [7]. Biémont et al. emphasized the importance of corepolarization effects on oscillator strength determination in Ce III [1]. The multiconfiguration Dirac-Fock calculation of the transition energies and oscillator strengths for the $6 s^{2}{ }^{1} \mathrm{~S}_{0}-6 s 6 p{ }^{1} \mathrm{P}_{1},{ }^{3} \mathrm{P}_{1}$ transitions in rare earth

*e-mail: bkaracoban@sakarya.edu.tr ionized systems (from $\mathrm{La}^{+1}$ through $\mathrm{Nd}^{+4}$ ) were carried out by Stanek and Migdałek [13].

Our aim here is to determine the radiative properties, such as wavelengths, logarithmic weighted oscillator strengths, and weighted transition probabilities, for electric dipole transitions (E1) in Ce III $(Z=58)$. These calculations have been performed by using code [14] developed Cowan for relativistic Hartree-Fock (HFR) calculations [15]. We have here investigated valence correlation effects and relativistic contributions. In this work, we considered different configuration sets for calculations according to valence correlation by the configuration interaction expansion. In calculations, we have taken into account two configuration sets represented with calculations $\mathrm{A}$ and $\mathrm{B}$, respectively:

$\mathrm{A}: 4 f^{2}, 5 d^{2}, 4 f n p(n=6,7), 4 f 5 f$, and $5 d 6 s$ (for even-parity); and $4 f n d(n=5-7), 4 f 6 s$, and $4 f 5 g$ (for odd-parity);

B: $4 f^{2}, 5 d^{2}, 4 f n p(n=6,7), 4 f n f(n=5,6), 5 d 6 s$, $5 d 6 d$, and $6 p^{2}$ (for even- parity); and $4 f n d(n=5-7)$, $4 f n s$ ( $n=6-8), 4 f 5 g$, and $5 d 6 p$ (for odd-parity).

In calculations, the core of $[\mathrm{Xe}]$ is fixed. These configuration sets used in calculations have been denoted by $\mathrm{A}$ and $\mathrm{B}$ in Table I and Table II. The energies, Landé $g$-factors, and lifetimes for $4 f^{2}, 4 f 5 d, 4 f 6 s, 5 d^{2}, 4 f 6 p$, $5 d 6 s, 4 f 6 d, 4 f 7 s, 5 d 6 p, 4 f 5 f, 4 f 7 p, 4 f 8 s, 4 f 7 d, 4 f 6 f$, $4 f 5 g, 6 p^{2}$, and $5 d 6 d$ excited levels of Ce III were presented in our other work [16]. In addition, we performed the atomic structure calculations on doubly ionized lanthanide and actinide atoms [17-21].

\section{Calculation method}

A detailed information on electromagnetic transition between two states can be found [15, 22]. Briefly, an electromagnetic transition between two states is characterized by the angular momentum and the parity of the corresponding photon. If the emitted or absorbed photon has angular momentum $k$ and parity $\pi=(-1)^{k}$ then, the transition is an electric multipole transition ( $\mathrm{E} k)$. However, if the photon has parity $\pi=(-1)^{k+1}$ the transition is a magnetic multipole transition $(\mathrm{M} k)$ [22]. 
The transition rate (or probability) for emission due to a transition from an upper level to a lower level is given by

$$
\begin{aligned}
& A^{\pi k}\left(\gamma^{\prime} J^{\prime}, \gamma J\right)= \\
& \quad 2 C_{k}\left[\alpha\left(E_{\gamma^{\prime} J^{\prime}}-E_{\gamma J}\right)\right]^{2 k+1} \frac{S^{\pi k}\left(\gamma^{\prime} J^{\prime}, \gamma J\right)}{g_{J^{\prime}}}
\end{aligned}
$$

and emission oscillator strength is given by

$$
\begin{aligned}
& f^{\pi k}\left(\gamma^{\prime} J^{\prime}, \gamma J\right)= \\
& \quad-\frac{1}{\alpha} C_{k}\left[\alpha\left(E_{\gamma J}-E_{\gamma^{\prime} J^{\prime}}\right)\right]^{2 k-1} \frac{S^{\pi k}\left(\gamma^{\prime} J^{\prime}, \gamma J\right)}{g_{J^{\prime}}},
\end{aligned}
$$

where $C_{k}=(2 k+1)(k+1) / k((2 k+1) ! !)^{2}$, and $g_{J^{\prime}}$ denotes the statistical weight of the upper level, namely $g_{J^{\prime}}=2 J^{\prime}+1$. In addition, $\alpha$ is the fine structure constant, and $\gamma$ denotes the configuration (seniority, configuration, coupling scheme, etc.). If each multipole is described by transition operator $O_{q}^{\pi(k)}$ (a spherical operator of rank $k$ and parity $\pi$ ), the line strength can be written as

$$
S^{\pi k}\left(\gamma J, \gamma^{\prime} J^{\prime}\right)=\sum_{M, M^{\prime}, q}|<\gamma J M| O_{q}^{\pi(k)}\left|\gamma^{\prime} J^{\prime} M^{\prime}>\right|^{2} .
$$

The strongest transition rate (or probability) is electric dipole (E1) radiation. For this reason, the E1 transitions are understood as being "allowed", whereas high-order transitions are understood as being "forbidden".

In HFR method, for $N$-electron atom of nuclear charge $Z_{0}$, the Hamiltonian is expanded as

$$
H=-\sum_{i} \nabla_{i}^{2}-\sum_{i} \frac{2 Z_{0}}{r_{i}}+\sum_{i>j} \frac{2}{r_{i j}}+\sum_{i} \zeta_{i}\left(r_{i}\right) \boldsymbol{l}_{i} \cdot \boldsymbol{s}_{i}
$$

in atomic units, with $r_{i}$ the distance of the $i$-th electron from the nucleus and $r_{i j}=\left|\boldsymbol{r}_{i}-\boldsymbol{r}_{j}\right| \cdot \zeta_{i}(R)=\frac{\alpha^{2}}{2} \frac{1}{r}\left(\frac{\partial V}{\partial r}\right)$ is the spin-orbit term, with $\alpha$ being the fine structure constant and $V$ the mean potential field due to the nucleus and other electrons.

In this method, one calculates single-configuration radial functions for a spherically symmetrised atom (centerof-gravity energy of the configuration) based on the Hartree-Fock method. The radial wave functions are also used to obtain the atom's total energy $\left(E_{\text {av }}\right)$ including approximate relativistic and correlation energy corrections. Relativistic terms in the potential function give approximate relativistic corrections to the radial functions, as well as improved relativistic energy corrections in heavy atoms. In addition, a correlation term is included to make the potential function more negative, thereby helping to bind negative ions. These radial functions are also used to calculate Coulomb integrals $F^{k}$ and $G^{k}$ and spinorbit integrals $\zeta_{n l}$. After radial functions have been obtained based on the Hartree-Fock model, the wave function $|\gamma J M\rangle$ of the $M$ sublevel of a level labeled $\gamma J$ is expressed in terms of $L S$ basis states $|\alpha L S J M\rangle$ by the formula

$$
|\gamma J M\rangle=\sum_{\alpha L S}|\alpha L S J M\rangle\langle\alpha L S J \mid \gamma J\rangle .
$$

If determinant wave functions are used for the atom, the total binding energy is given by

$$
E=\sum_{i}\left(E_{k}^{i}+E_{n}^{i}+\sum_{j<i} E^{i j}\right),
$$

where $E_{k}^{i}$ is the kinetic energy, $E_{n}^{i}$ is the electron-nuclear Coulomb energy, and $E^{i j}$ is the Coulomb interaction energy between electrons $i$ and $j$ averaged over all possible magnetic quantum numbers.

In this method, relativistic corrections have been limited to calculations to the mass-velocity and the Darwin corrections by using the relativistic correction to total binding energy. The total binding energy can be given by formulae (7.57), (7.58), and (7.59) in [15].

\section{Results and discussion}

In our previous work, we presented the relativistic energies, Landé $g$-factors, and lifetimes for $4 f^{2}, 4 f 5 d, 4 f 6 s$, $5 d^{2}, 4 f 6 p, 5 d 6 s, 4 f 6 d, 4 f 7 s, 5 d 6 p, 4 f 5 f, 4 f 7 p, 4 f 8 s$, $4 f 7 d, 4 f 6 f, 4 f 5 g, 6 p^{2}$, and $5 d 6 d$ excited levels outside the core [Xe] in Ce III [16]. In this work, we have calculated the transition parameters (wavelengths, oscillator strengths, and transition probabilities) for electric dipole (E1) transitions in Ce III using HFR code [14]. Two different calculations have been here performed to obtain configuration state functions (CSFs) according to valence correlation. The results obtained from this work have been reported in Tables I and II. In these tables, the calculations for the two configuration sets are represented by A and B. In Table I, a comparison with other calculations and experiments has been made. References for other comparison values are typed below the Table I with a superscript lower letter. Only odd-parity levels are indicated by the superscript "o". Tables I and II include only a part of the large-scale transition calculations.

In calculations, the Hamiltonian's calculated eigenvalues were optimized to the observed energy levels via a least-squares fitting procedure using experimentally determined energy levels, specifically all of the levels from the NIST compilation [23]. The scaling factors of the Slater parameters $\left(F^{k}\right.$ and $\left.G^{k}\right)$ and of configuration interaction integrals $\left(R^{k}\right)$, not optimized in the leastsquares fitting, were chosen equal to 0.75 for calculations $\mathrm{A}$ and $\mathrm{B}$, while the spin-orbit parameters were left at their initial values. This low value of the scaling factors has been suggested by Cowan for heavy elements $[14,15]$.

We obtained 3096 and 6216 possible electric dipole transitions between even- and odd-parity levels in the calculations $\mathrm{A}$ and $\mathrm{B}$, respectively. Table I shows the wavelengths, $\lambda$ (in $\AA$ ), the logarithmic weighted oscillator strengths, $\log (g f)$, and the weighted transition rates (or probabilities), $g A_{k i}$ (in $\mathrm{s}^{-1}$ ), for $4 f^{2}-4 f 5 d$, $4 f^{2}-4 f 6 d, 4 f^{2}-4 f 6 s, 4 f 5 d-5 d^{2}, 4 f 5 d-5 d 6 s, 4 f 5 d-4 f 6 p$, $4 f 5 d-4 f 5 f, 4 f 6 s-5 d^{2}, 4 f 6 s-4 f 6 p$ and $4 f 6 s-5 d 6 s$ electric dipole transitions. The comparing values for these transitions exist in literature. Therefore, it is also made a comparison with other works in Table I. We have presented the best values obtained from $\mathrm{A}$ and $\mathrm{B}$ configuration sets for Ce III in Table I. Energy of levels in this table can be found in our previous work [16]. In addition, we have reported the wavelengths, the logarithmic weighted oscillator strengths, $\log (g f)$, and the 
weighted transition probabilities, $g A_{k i}$, for some transitions in Table II (all data can be obtained from corresponding author). In Table II, the lower and upper levels of each transition are presented by their $J$-values, parities [(e) for even and (o) for odd], and energy calculation results $\left[E_{t h}, 16\right]$ and experimental values $\left[E_{\exp }, 23\right]$. Biémont and co-workers indicated that a more extensive table (3000 transitions) of $g f$ - and $g A$-values computed in their work is available in the DREAM database on the web at http://www.umh.ac.be/ astro/dream.shtml [1]. But we have not reached that data from this web address. Therefore, the compared values for transitions in Table II may exist in this web site.

In Table I, except some transitions an agreement is seen when our results are compared with other works $[1,6,7,12]$. Although the agreement is less in the weighted transition probabilities, it is good in the wavelengths and the logarithmic weighted oscillator strengths. For weighted transition probabilities of $4 f 5 d{ }^{3} G_{4}^{o}-4 f\left({ }^{2} F_{7 / 2}^{o}\right) 6 p_{1 / 2} \quad(7 / 2$, $1 / 2)_{3}, 4 f 5 d{ }^{1} F_{3}^{o}-4 f\left({ }^{2} F_{5 / 2}^{o}\right) 6 p_{3 / 2}(5 / 2,3 / 2)_{4}, 4 f 5 d{ }^{1} H_{5}^{o}-$ $4 f\left({ }^{2} F_{7 / 2}^{o}\right) 6 p_{3 / 2}(7 / 2,3 / 2)_{4}, 4 f\left({ }^{2} F_{7 / 2}^{o}\right) 6 s_{1 / 2}(7 / 2,1 / 2)_{3}^{o}$ $5 d^{2}{ }^{3} F_{4}$ and $4 f\left({ }^{2} F_{7 / 2}^{o}\right) 6 s_{1 / 2}(7 / 2,1 / 2)_{4}^{o}-5 d^{2}{ }^{3} F_{4}$ transitions, the agreement is poor. Of course, these results from HFR calculations will be better in case that the configurations including the excitations from $5 p^{6}$ are added that is, considering the core correlation which is from excitations core subshells. But this case occurs some program constraints or convergence problems. In addition, we have given a comparison in Fig. 1 for logarithmic weighted oscillator strengths obtained from HFR calculations with those in works $[1,6,7]$. As seen from Fig. 1, the $\log (g f)$ obtained from our calculations are in good agreement with $[1,6,7]$.

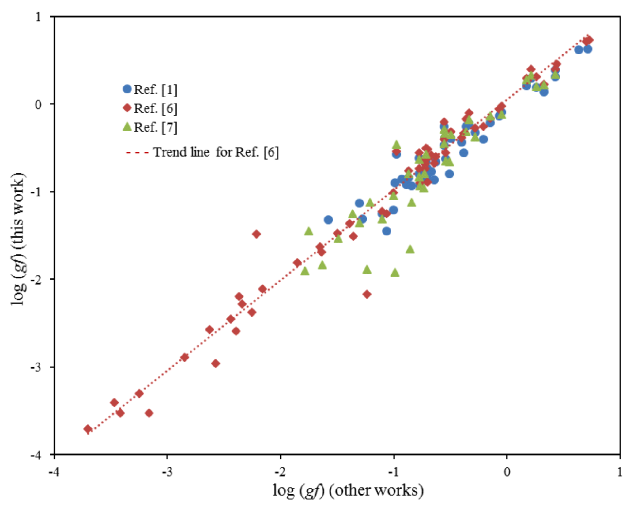

Fig. 1. Comparison of the logarithmic oscillator strengths obtained from this work with those of Biémont et al. [1], Wyart and Palmeri [6] and $\mathrm{Li}$ et al. [7].

It is well known that the correlation, relativistic and radiative effects all play important roles in fundamental atomic theory. The main purpose of this paper is to perform the Hartree-Fock calculations for obtaining description of the Ce III spectrum. Especially, in spectrum synthesis works, particularly for CP stars, accurate data for transition parameters for lanthanides are need to establish reliable abundances for species. Therefore we hope that our results obtained using HFR method will be useful for research fields and technological applications and for interpreting the spectrum of Ce III.

\section{Acknowledgments}

The author is very grateful to the anonymous reviewer for stimulating comments and valuable suggestions, which is resulted in improving the presentation of the paper.

\section{References}

[1] E. Biémont, P. Quinet, T.A. Ryabchikova, Mon. Not. R. Astron. Soc. 336, 1155 (2002).

[2] J.J. Curry, J. Phys. D Appl. Phys. 42, 135205 (2009).

[3] N. Spector, J. Opt. Soc. Am. 55, 492 (1965).

[4] T. Andersen, G. Sørensen, Solar Phys. 38, 343 (1974).

[5] P. Quinet, E. Biémont, At. Data Nucl. Data Tables 87, 207 (2004).

[6] J.-F. Wyart, P. Palmeri, Phys. Scr. 58, 368 (1998).

[7] Z.S. Li, H. Lundberg, G.M. Wahlgren, C.M. Sikström, Phys. Rev. A 62, 032505 (2000).

[8] A.S. King, R.B. King, Astrophys. J. 75, 40 (1932).

[9] H.N. Russell, R.B. King, R.J. Lang, Phys. Rev. 52, 456 (1937).

[10] J. Sugar, J. Opt. Soc. Am. 55, 33 (1965).

[11] S. Johansson, U. Litzén, Phys. Scr. 6, 139 (1972).

[12] D.J. Bord, C.R Cowley, P.L. Norquist, Mon. Not. R. Astron. Soc. 284, 869 (1997).

[13] M. Stanek, J. Migdałek, J. Phys. B At. Mol. Opt. Phys. 37, 2707 (2004).

[14] http://www.tcd.ie/Physics/People/ Cormac.McGuinness/Cowan/.

[15] R.D. Cowan, The Theory of Atomic Structure Spectra, University of California Press, USA 1981.

[16] B. Karaçoban Usta, Chin. J. Phys. 53, 040401-1 (2015).

[17] B. Karaçoban, L. Özdemir, Cent. Eur. J. Phys. 10, 124 (2012).

[18] B. Karaçoban, L. Özdemir, J. At. Mol. Opt. Phys. 2012, 1 (2012).

[19] B. Karaçoban, L. Özdemir, Acta Phys. Pol. A 123, 651 (2013).

[20] G. Ürer, B. Karaçoban, L. Özdemir, $B P L$ 21, 90 (2013).

[21] B. Karaçoban, L. Özdemir, J. Phys. Ast. 3, 10 (2014).

[22] C.F. Fischer, T. Brage, P. Jönsson, Computational Atomic Structure - An MCHF Approach, IOP, Bristol 1997.

[23] http://www.nist.gov/physlab/data/asd.cfm. 
TABLE I

Wavelengths, $\lambda$, logarithmic weighted oscillator strengths, $\log (g f)$, and weighted transition probabilities, $g A_{k i}$, for electric dipole (E1) transitions in Ce III.

\begin{tabular}{|c|c|c|c|c|c|c|c|c|c|}
\hline \multicolumn{4}{|c|}{ Transition } & \multicolumn{2}{|c|}{$\overline{\lambda \lambda[\AA]}$} & \multicolumn{2}{|c|}{$\log (g f)$} & \multicolumn{2}{|c|}{$g A_{k i}\left[\mathrm{~s}^{-1}\right]$} \\
\hline \multicolumn{2}{|c|}{ Lower Level } & \multicolumn{2}{|c|}{ Upper level } & This & Other & This & Other & This & Other \\
\hline $4 f 5 d$ & ${ }^{3} H_{6}^{o}$ & $4 f\left({ }^{2} F_{7 / 2}^{o}\right) 5 f$ & ${ }^{2}[13 / 2]_{7}$ & $1068.648^{\mathrm{B}}$ & $\begin{array}{l}1072.791^{a} \\
1072.790^{b}\end{array}$ & $\begin{array}{l}0.635^{\mathrm{A}} \\
0.697^{\mathrm{B}}\end{array}$ & $\begin{array}{c}0.62^{a 1} \\
0.72^{a 2, b}\end{array}$ & $2.90 \times 10^{10 B}$ & $3.027 \times 10^{10 b}$ \\
\hline $4 f^{2}$ & ${ }^{3} P_{2}$ & $4 f\left({ }^{2} F_{7 / 2}^{o}\right) 6 d$ & ${ }^{2}[3 / 2]_{2}^{o}$ & $1325.101^{\mathrm{A}}$ & $1324.893^{b}$ & $\begin{array}{l}-2.190^{\mathrm{A}} \\
-1.983^{\mathrm{B}}\end{array}$ & - & $2.45 \times 10^{7 A}$ & $1.101 \times 10^{7 b}$ \\
\hline $4 f 5 d$ & ${ }^{3} D_{2}^{o}$ & $5 d 6 s$ & ${ }^{3} D_{2}$ & $1849.122^{\mathrm{A}}$ & $1848.088^{b}$ & $\begin{array}{l}-1.398^{\mathrm{A}} \\
-1.436^{\mathrm{B}}\end{array}$ & - & $7.18 \times 10^{7 B}$ & $6.811 \times 10^{7 b}$ \\
\hline $4 f 5 d$ & ${ }^{1} G_{4}^{o}$ & $4 f\left({ }^{2} F_{7 / 2}^{o}\right) 6 p_{3 / 2}$ & $(7 / 2,3 / 2)_{4}$ & $1958.322^{\mathrm{B}}$ & $\begin{array}{l}1950.356^{a} \\
1950.355^{b}\end{array}$ & $-0.773^{\mathrm{A}}$ & $\begin{array}{c}-0.92^{a 1} \\
-0.90^{a 2, b} \\
-0.93^{a 3, c}\end{array}$ & $2.89 \times 10^{8 A}$ & $\begin{array}{c}2.182 \times 10^{8 b} \\
2.43 \times 10^{8 c}\end{array}$ \\
\hline $4 f 5 d$ & ${ }^{1} G_{4}^{o}$ & $4 f\left({ }^{2} F_{7 / 2}^{o}\right) 6 p_{3 / 2}$ & $(7 / 2,3 / 2)_{3}$ & $1978.172^{\mathrm{B}}$ & $1986.519^{a}$ & $\begin{array}{l}-1.209^{\mathrm{A}} \\
-1.276^{\mathrm{B}}\end{array}$ & $\begin{array}{c}-1.31^{a 1} \\
-1.12^{a 2, c}\end{array}$ & $1.01 \times 10^{8 A}$ & $1.50 \times 10^{8 c}$ \\
\hline $4 f 5 d$ & ${ }^{1} P_{1}^{o}$ & $5 d^{2}$ & ${ }^{1} S_{0}$ & $2027.139^{\mathrm{B}}$ & $2028.293^{a, b}$ & $-0.647^{\mathrm{B}}$ & $\begin{array}{c}-0.86^{a 1} \\
-0.68^{a 2} \\
-0.67^{b}\end{array}$ & $3.66 \times 10^{8 B}$ & $3.422 \times 10^{8 b}$ \\
\hline $4 f 5 d$ & ${ }^{3} F_{3}^{o}$ & $4 f\left({ }^{2} F_{7 / 2}^{o}\right) 6 p_{3 / 2}$ & $(7 / 2,3 / 2)_{4}$ & $2042.571^{\mathrm{B}}$ & $2038.207^{c}$ & $-1.632^{\mathrm{A}}$ & $-1.83^{c}$ & $3.68 \times 10^{7 A}$ & $2.82 \times 10^{7 c}$ \\
\hline $4 f 5 d$ & ${ }^{3} F_{3}^{o}$ & $4 f\left({ }^{2} F_{7 / 2}^{o}\right) 6 p_{3 / 2}$ & $(7 / 2,3 / 2)_{3}$ & $2064.174^{\mathrm{B}}$ & $2077.752^{c}$ & $-1.787^{\mathrm{A}}$ & $-1.90^{c}$ & $2.45 \times 10^{7 A}$ & $2.30 \times 10^{7 c}$ \\
\hline $4 f 5 d$ & ${ }^{3} F_{4}^{o}$ & $4 f\left({ }^{2} F_{7 / 2}^{o}\right) 6 p_{3 / 2}$ & $(7 / 2,3 / 2)_{4}$ & $2119.771^{\mathrm{B}}$ & $\begin{array}{c}2109.068^{a, c} \\
2109.066^{b}\end{array}$ & $-0.556^{\mathrm{B}}$ & $\begin{array}{c}-0.47^{a 1} \\
-0.40^{a 2, b} \\
-0.45^{a 3, c}\end{array}$ & $4.13 \times 10^{8 B}$ & $\begin{array}{c}6.015 \times 10^{8 b} \\
6.35 \times 10^{8 c}\end{array}$ \\
\hline $4 f 5 d$ & ${ }^{3} F_{2}^{o}$ & $4 f\left({ }^{2} F_{7 / 2}^{o}\right) 6 p_{1 / 2}$ & $(7 / 2,1 / 2)_{3}$ & $2156.077^{\mathrm{B}}$ & $2147.392^{c}$ & $-0.857^{\mathrm{B}}$ & $-1.65^{c}$ & $2.13 \times 10^{8 A}$ & $3.93 \times 10^{7 c}$ \\
\hline $4 f 5 d$ & ${ }^{3} F_{4}^{O}$ & $4 f\left({ }^{2} F_{7 / 2}^{o}\right) 6 p_{3 / 2}$ & $(7 / 2,3 / 2)_{3}$ & $2143.048^{\mathrm{B}}$ & $2151.438^{a, b}$ & $-0.552^{\mathrm{B}}$ & $\begin{array}{c}-0.38^{a 1} \\
-0.31^{a 2, b} \\
-0.33^{a 3, c}\end{array}$ & $4.07 \times 10^{8 B}$ & $\begin{array}{c}6.992 \times 10^{8 b} \\
7.98 \times 10^{8 c}\end{array}$ \\
\hline $4 f 5 d$ & ${ }^{3} H_{6}^{o}$ & $4 f\left({ }^{2} F_{7 / 2}^{o}\right) 6 p_{3 / 2}$ & $(7 / 2,3 / 2)_{5}$ & $2189.023^{\mathrm{A}}$ & $2180.635^{a, b}$ & $\begin{array}{l}0.427^{\mathrm{A}} \\
0.438^{\mathrm{B}}\end{array}$ & $\begin{array}{c}0.39^{a 1} \\
0.46^{a 2, b}\end{array}$ & $3.79 \times 10^{9 B}$ & $4.031 \times 10^{9 b}$ \\
\hline $4 f 5 d$ & ${ }^{3} F_{3}^{o}$ & $4 f\left({ }^{2} F_{5 / 2}^{o}\right) 6 p_{3 / 2}$ & $(5 / 2,3 / 2)_{3}$ & $2176.844^{\mathrm{A}}$ & $2184.639^{a, c}$ & $-0.509^{\mathrm{A}}$ & $\begin{array}{c}-0.79^{a 1} \\
-0.66^{a 2, c}\end{array}$ & $4.36 \times 10^{8 A}$ & $3.81 \times 10^{8 c}$ \\
\hline $4 f 5 d$ & ${ }^{3} G_{3}^{o}$ & $4 f\left({ }^{2} F_{5 / 2}^{o}\right) 6 p_{3 / 2}$ & $(5 / 2,3 / 2)_{3}$ & $2200.584^{\mathrm{A}}$ & $2221.679^{a, c}$ & $-1.307^{\mathrm{B}}$ & $\begin{array}{c}-1.13^{a 1} \\
-1.35^{a 2, c}\end{array}$ & $7.24 \times 10^{7 B}$ & $7.37 \times 10^{7 c}$ \\
\hline $4 f 5 d$ & ${ }^{3} F_{3}^{o}$ & $4 f\left({ }^{2} F_{7 / 2}^{o}\right) 6 p_{1 / 2}$ & $(7 / 2,1 / 2)_{3}$ & $2234.914^{\mathrm{B}}$ & $\begin{array}{l}2227.837^{a} \\
2227.835^{b}\end{array}$ & $-0.281^{\mathrm{A}}$ & $\begin{array}{c}-0.32^{a 1} \\
-0.27^{a 2, b} \\
-0.37^{a 3, c}\end{array}$ & $6.87 \times 10^{8 A}$ & $\begin{array}{l}7.180 \times 10^{8 b} \\
6.97 \times 10^{8 c}\end{array}$ \\
\hline $4 f 5 d$ & ${ }^{3} G_{5}^{o}$ & $4 f\left({ }^{2} F_{7 / 2}^{o}\right) 6 p_{3 / 2}$ & $(7 / 2,3 / 2)_{5}$ & $2242.072^{\mathrm{A}}$ & $\begin{array}{l}2228.051^{a} \\
2228.050^{b}\end{array}$ & $-0.069^{\mathrm{B}}$ & $\begin{array}{c}-0.13^{a 1} \\
-0.06^{a 2, b}\end{array}$ & $1.14 \times 10^{9 A}$ & $1.175 \times 10^{9 b}$ \\
\hline $4 f 5 d$ & ${ }^{3} F_{2}^{o}$ & $4 f\left({ }^{2} F_{5 / 2}^{o}\right) 6 p_{1 / 2}$ & $(5 / 2,1 / 2)_{2}$ & $2261.955^{\mathrm{A}}$ & $\begin{array}{l}2242.295^{a} \\
2242.293^{b}\end{array}$ & $-0.361^{\mathrm{B}}$ & $\begin{array}{c}-0.25^{a 1} \\
-0.17^{a 2, b} \\
-0.31^{a 3, c}\end{array}$ & $5.95 \times 10^{8 B}$ & $\begin{array}{c}8.902 \times 10^{8 b} \\
7.51 \times 10^{8 c}\end{array}$ \\
\hline $4 f\left({ }^{2} F_{5 / 2}^{o}\right) 6 s_{1 / 2}$ & $(5 / 2,1 / 2)_{2}^{o}$ & $5 d 6 s$ & ${ }^{3} D_{1}$ & $2267.110^{\mathrm{A}}$ & $\begin{array}{l}2266.915^{a} \\
2266.914^{b}\end{array}$ & $-0.210^{\mathrm{B}}$ & $\begin{array}{c}-0.40^{a 1} \\
-0.25^{a 2, b}\end{array}$ & $8.08 \times 10^{8 B}$ & $7.342 \times 10^{8 b}$ \\
\hline $4 f 5 d$ & ${ }^{3} D_{3}^{o}$ & $4 f\left({ }^{2} F_{7 / 2}^{o}\right) 6 p_{3 / 2}$ & $(7 / 2,3 / 2)_{3}$ & $2279.834^{\mathrm{B}}$ & $2298.700^{a}$ & $-0.989^{\mathrm{A}}$ & $\begin{array}{l}-0.90^{a 1} \\
-1.92^{a 2, c}\end{array}$ & $1.26 \times 10^{8 A}$ & $1.78 \times 10^{7 c}$ \\
\hline $4 f 5 d$ & ${ }^{3} H_{4}^{o}$ & $4 f\left({ }^{2} F_{5 / 2}^{o}\right) 6 p_{1 / 2}$ & $(5 / 2,1 / 2)_{3}$ & $2303.238^{\mathrm{B}}$ & $\begin{array}{c}2317.337^{a, c} \\
2317.338^{b}\end{array}$ & $-0.981^{\mathrm{B}}$ & $\begin{array}{c}-0.57^{a 1} \\
-0.54^{a 2, b} \\
-0.46^{a 3, c}\end{array}$ & $1.31 \times 10^{8 B}$ & $\begin{array}{c}3.552 \times 10^{8 b} \\
5.17 \times 10^{8 c}\end{array}$ \\
\hline $4 f 5 d$ & ${ }^{3} G_{5}^{o}$ & $4 f\left({ }^{2} F_{5 / 2}^{o}\right) 6 p_{3 / 2}$ & $(5 / 2,3 / 2)_{4}$ & $2410.457^{\mathrm{A}}$ & $\begin{array}{c}2318.642^{a, c} \\
2318.643^{b}\end{array}$ & $-0.049^{\mathrm{A}}$ & $\begin{array}{c}-0.09^{a 1} \\
-0.02^{a 2, b} \\
-0.12^{a 3, c}\end{array}$ & $1.03 \times 10^{9 A}$ & $\begin{array}{c}1.196 \times 10^{9 b} \\
1.17 \times 10^{9 c}\end{array}$ \\
\hline $4 f 5 d$ & ${ }^{3} D_{1}^{o}$ & $4 f\left({ }^{2} F_{5 / 2}^{o}\right) 6 p_{3 / 2}$ & $(5 / 2,3 / 2)_{1}$ & $2384.821^{\mathrm{B}}$ & $\begin{array}{l}2324.311^{a} \\
2324.310^{b}\end{array}$ & $-0.632^{\mathrm{B}}$ & $\begin{array}{c}-0.66^{a 1} \\
-0.60^{a 2, b}\end{array}$ & $2.73 \times 10^{8 B}$ & $3.139 \times 10^{8 b}$ \\
\hline $4 f 5 d$ & ${ }^{3} F_{3}^{o}$ & $4 f\left({ }^{2} F_{5 / 2}^{o}\right) 6 p_{1 / 2}$ & $(5 / 2,1 / 2)_{3}$ & $2327.994^{\mathrm{B}}$ & $2337.664^{a, c}$ & $-0.847^{\mathrm{A}}$ & $\left|\begin{array}{c}-0.93^{a 1} \\
-1.12^{a 2, c}\end{array}\right|$ & $1.70 \times 10^{8 A}$ & $1.12 \times 10^{8 c}$ \\
\hline
\end{tabular}


TABLE I (cont.)

Wavelengths, $\lambda$, logarithmic weighted oscillator strengths, $\log (g f)$, and weighted transition probabilities, $g A_{k i}$, for electric dipole (E1) transitions in Ce III.

\begin{tabular}{|c|c|c|c|c|c|c|c|c|c|}
\hline \multicolumn{4}{|c|}{ Transition } & \multicolumn{2}{|c|}{$\overline{\bar{\lambda} \lambda[\AA]}$} & \multicolumn{2}{|c|}{$\log (g f)$} & \multicolumn{2}{|c|}{$g A_{k i}\left[\mathrm{~s}^{-1}\right]$} \\
\hline \multicolumn{2}{|c|}{ Lower Level } & \multicolumn{2}{|c|}{ Upper level } & This & Other & This & Other & This & Other \\
\hline $4 f 5 d$ & ${ }^{3} G_{4}^{o}$ & $4 f\left({ }^{2} F_{7 / 2}^{o}\right) 6 p_{1 / 2}$ & $(7 / 2,1 / 2)_{3}$ & $2353.809^{\mathrm{B}}$ & $2350.104^{a, b, c}$ & $-0.556^{\mathrm{A}}$ & $\begin{array}{l}-0.25^{a 1} \\
-0.20^{a 2, b} \\
-0.29^{a 3, c}\end{array}$ & $3.30 \times 10^{8 A}$ & $\begin{array}{c}7.666 \times 10^{8 b} \\
7.44 \times 10^{8 c}\end{array}$ \\
\hline $4 f 5 d$ & ${ }^{3} D_{3}^{o}$ & $4 f\left({ }^{2} F_{5 / 2}^{o}\right) 6 p_{3 / 2}$ & $(5 / 2,3 / 2)_{4}$ & $2297.706^{\mathrm{B}}$ & $2362.538^{a, c}$ & $-0.739^{\mathrm{B}}$ & $\begin{array}{c}-0.85^{a 1} \\
-0.96^{a 2, c}\end{array}$ & $2.31 \times 10^{8 B}$ & $1.63 \times 10^{8 c}$ \\
\hline $4 f 5 d$ & ${ }^{1} F_{3}^{o}$ & $4 f\left({ }^{2} F_{7 / 2}^{o}\right) 6 p_{3 / 2}$ & $(7 / 2,3 / 2)_{2}$ & $2361.665^{\mathrm{A}}$ & $\begin{array}{l}2377.070^{a} \\
2377.071^{b}\end{array}$ & $-0.730^{\mathrm{A}}$ & $\begin{array}{c}-0.66^{a 1} \\
-0.62^{a 2, b}\end{array}$ & $2.23 \times 10^{8 A}$ & $2.849 \times 10^{8 b}$ \\
\hline $4 f 5 d$ & ${ }^{1} F_{3}^{o}$ & $4 f\left({ }^{2} F_{7 / 2}^{o}\right) 6 p_{3 / 2}$ & $(7 / 2,3 / 2)_{4}$ & $2391.678^{\mathrm{B}}$ & $\begin{array}{l}2377.474^{a} \\
2377.475^{b}\end{array}$ & $-0.871^{\mathrm{A}}$ & $\begin{array}{c}-0.84^{a 1} \\
-0.76^{a 2, b} \\
0.703, c\end{array} \mid$ & $1.54 \times 10^{8 A}$ & $\begin{array}{c}2.077 \times 10^{8 b} \\
2.28 \times 10^{8 c}\end{array}$ \\
\hline $4 f 5 d$ & ${ }^{3} D_{2}^{o}$ & $4 f\left({ }^{2} F_{5 / 2}^{o}\right) 6 p_{3 / 2}$ & $(5 / 2,3 / 2)_{2}$ & $2372.245^{\mathrm{B}}$ & $2395.042^{b}$ & $-0.732^{\mathrm{A}}$ & $\mid \begin{array}{c}-0.79^{a s, c} \\
-0.66^{b} \\
-0.79^{c}\end{array}$ & $2.02 \times 10^{8 A}$ & $\begin{array}{c}2.555 \times 10^{8 b} \\
2.21 \times 10^{8 c}\end{array}$ \\
\hline $4 f 5 d$ & ${ }^{1} D_{2}^{o}$ & $4 f\left({ }^{2} F_{5 / 2}^{o}\right) 6 p_{1 / 2}$ & $(5 / 2,1 / 2)_{3}$ & $2417.807^{\mathrm{B}}$ & $2397.602^{c}$ & $-1.366^{\mathrm{B}}$ & $-1.25^{c}$ & $4.91 \times 10^{7 B}$ & $7.93 \times 10^{7 c}$ \\
\hline $4 f 5 d$ & ${ }^{1} F_{3}^{o}$ & $4 f\left({ }^{2} F_{7 / 2}^{o}\right) 6 p_{3 / 2}$ & $(7 / 2,3 / 2)_{3}$ & $2421.351^{\mathrm{B}}$ & $2431.449^{a, b, c}$ & $-0.151^{\mathrm{A}}$ & $\begin{array}{l}-0.21^{a 1} \\
-0.16^{a 2, b} \\
-0.13^{a 3, c}\end{array}$ & $7.64 \times 10^{8 A}$ & $\begin{array}{l}7.846 \times 10^{8 b} \\
9.88 \times 10^{8 c}\end{array}$ \\
\hline $4 f 5 d$ & ${ }^{3} G_{4}^{o}$ & $4 f\left({ }^{2} F_{5 / 2}^{o}\right) 6 p_{1 / 2}$ & $(5 / 2,1 / 2)_{3}$ & $2487.577^{\mathrm{A}}$ & $2472.646^{c}$ & $-1.490^{\mathrm{A}}$ & $-1.53^{c}$ & $3.49 \times 10^{7 A}$ & $3.89 \times 10^{7 c}$ \\
\hline $4 f 5 d$ & ${ }^{3} P_{0}^{o}$ & $4 f\left({ }^{2} F_{5 / 2}^{o}\right) 6 p_{3 / 2}$ & $(5 / 2,3 / 2)_{1}$ & $2554.093^{\mathrm{B}}$ & $\begin{array}{l}2477.248^{a} \\
2477.249^{b}\end{array}$ & $-0.729^{\mathrm{B}}$ & $\begin{array}{l}-0.79^{a 1} \\
-0.73^{a 2, b}\end{array}$ & $1.91 \times 10^{8 B}$ & $2.038 \times 10^{8 b}$ \\
\hline $4 f 5 d$ & ${ }^{3} P_{1}^{o}$ & $4 f\left({ }^{2} F_{5 / 2}^{o}\right) 6 p_{3 / 2}$ & $(5 / 2,3 / 2)_{1}$ & $2552.171^{\mathrm{B}}$ & $\begin{array}{l}2479.430^{a} \\
2479.437^{b}\end{array}$ & $-0.707^{\mathrm{B}}$ & $\begin{array}{c}-0.80^{a 1} \\
-0.74^{a 2} \\
-0.89^{b}\end{array}$ & $2.01 \times 10^{8 B}$ & $1.996 \times 10^{8 b}$ \\
\hline $4 f 5 d$ & ${ }^{3} P_{1}^{o}$ & $4 f\left({ }^{2} F_{5 / 2}^{o}\right) 6 p_{3 / 2}$ & $(5 / 2,3 / 2)_{2}$ & $2582.774^{\mathrm{A}}$ & $\begin{array}{c}2497.498^{a, c} \\
2497.497^{b}\end{array}$ & $-0.780^{\mathrm{B}}$ & $\begin{array}{c}-0.61^{a 1} \\
-0.55^{a 2, b}\end{array}$ & $1.56 \times 10^{8 B}$ & $\begin{array}{c}3.025 \times 10^{8 b} \\
2.93 \times 10^{8 c}\end{array}$ \\
\hline $4 f 5 d$ & ${ }^{1} F_{3}^{o}$ & $4 f\left({ }^{2} F_{5 / 2}^{o}\right) 6 p_{3 / 2}$ & $(5 / 2,3 / 2)_{4}$ & $2441.520^{\mathrm{B}}$ & $2502.986^{c}$ & $-1.755^{\mathrm{A}}$ & $\begin{array}{c}-0.63^{a 3, c} \\
-1.45^{c}\end{array}$ & $1.69 \times 10^{7 A}$ & $4.67 \times 10^{7 c}$ \\
\hline $4 f 5 d$ & ${ }^{3} D_{3}^{o}$ & $4 f\left({ }^{2} F_{7 / 2}^{o}\right) 6 p_{1 / 2}$ & $(7 / 2,1 / 2)_{4}$ & $2446.755^{\mathrm{B}}$ & $\begin{array}{c}2503.561^{a, c} \\
2503.562^{b}\end{array}$ & $-1.106^{\mathrm{A}}$ & $\begin{array}{l}-1.25^{a 1} \\
-1.22^{a 2, b} \\
-1.31^{a 3, c}\end{array}$ & $9.47 \times 10^{7 A}$ & $\begin{array}{c}6.363 \times 10^{7 b} \\
6.10 \times 10^{7 c}\end{array}$ \\
\hline $4 f 5 d$ & ${ }^{3} D_{1}^{o}$ & $4 f\left({ }^{2} F_{5 / 2}^{o}\right) 6 p_{1 / 2}$ & $(5 / 2,1 / 2)_{2}$ & $2544.001^{\mathrm{A}}$ & $\begin{array}{c}2531.987^{a, c} \\
2531.986^{b}\end{array}$ & $-0.544^{\mathrm{A}}$ & $\begin{array}{c}-0.62^{a 1} \\
-0.55^{a 2, b} \\
-0.65^{a 3, c}\end{array}$ & $2.95 \times 10^{8 A}$ & $\begin{array}{c}2.951 \times 10^{8 b} \\
2.67 \times 10^{8 c}\end{array}$ \\
\hline $4 f 5 d$ & ${ }^{1} H_{5}^{o}$ & $4 f\left({ }^{2} F_{7 / 2}^{o}\right) 6 p_{3 / 2}$ & $(7 / 2,3 / 2)_{4}$ & $2615.306^{\mathrm{B}}$ & $\begin{array}{c}2603.591^{a, c} \\
2603.590^{b}\end{array}$ & $0.423^{\mathrm{B}}$ & $\begin{array}{c}0.31^{a 1} \\
0.40^{a 2, b} \\
0.34^{a 3, c}\end{array}$ & $2.58 \times 10^{9 B}$ & $\begin{array}{c}2.456 \times 10^{9 b} \\
2.59 \times 10^{9 c}\end{array}$ \\
\hline $4 f 5 d$ & ${ }^{3} D_{3}^{o}$ & $5 d^{2}$ & ${ }^{1} D_{2}$ & $2719.320^{\mathrm{B}}$ & $\begin{array}{l}2719.301^{a} \\
2719.297^{b}\end{array}$ & $-0.673^{\mathrm{A}}$ & $\begin{array}{l}-0.77^{a 1} \\
-0.58^{a 2, b}\end{array}$ & $2.53 \times 10^{8 B}$ & $3.257 \times 10^{8 b}$ \\
\hline $4 f 5 d$ & ${ }^{3} F_{3}^{o}$ & $5 d^{2}$ & ${ }^{3} F_{3}$ & $2742.050^{\mathrm{A}}$ & $\begin{array}{l}2743.714^{a} \\
2743.713^{b}\end{array}$ & $\begin{array}{l}-0.721^{\mathrm{A}} \\
-0.891^{\mathrm{B}}\end{array}$ & $\begin{array}{c}-0.91^{a 1} \\
-0.73^{a 2, b}\end{array}$ & $1.69 \times 10^{8 A}$ & $1.633 \times 10^{8 b}$ \\
\hline $4 f 5 d$ & ${ }^{3} F_{4}^{o}$ & $5 d^{2}$ & ${ }^{3} F_{4}$ & $2740.456^{\mathrm{A}}$ & $2748.902^{a}$ & $\begin{array}{l}-0.727^{\mathrm{A}} \\
-0.934^{\mathrm{B}}\end{array}$ & $\begin{array}{c}-0.85^{a 1} \\
-0.68^{a 2, b}\end{array}$ & $1.67 \times 10^{8 A}$ & $1.843 \times 10^{8 b}$ \\
\hline $4 f 5 d$ & ${ }^{1} H_{5}^{o}$ & $4 f\left({ }^{2} F_{5 / 2}^{o}\right) 6 p_{3 / 2}$ & $(5 / 2,3 / 2)_{4}$ & $2888.357^{\mathrm{A}}$ & $2754.869^{a, c}$ & $-0.717^{\mathrm{A}}$ & $\begin{array}{l}-0.66^{a 1} \\
-0.50^{a 2, b} \\
-0.57^{a 3, c}\end{array}$ & $1.54 \times 10^{8 A}$ & $\begin{array}{c}2.762 \times 10^{8 b} \\
2.95 \times 10^{8 c}\end{array}$ \\
\hline $4 f 5 d$ & ${ }^{1} P_{1}^{o}$ & $4 f\left({ }^{2} F_{7 / 2}^{o}\right) 6 p_{3 / 2}$ & $(7 / 2,3 / 2)_{2}$ & $2730.164^{\mathrm{A}}$ & $2768.280^{a}$ & $-0.402^{\mathrm{B}}$ & $\begin{array}{c}-0.43^{a 1} \\
-0.38^{a 2, b}\end{array}$ & $3.32 \times 10^{8 B}$ & $3.662 \times 10^{8 b}$ \\
\hline $4 f 5 d$ & ${ }^{1} F_{3}^{o}$ & $4 f\left({ }^{2} F_{5 / 2}^{o}\right) 6 p_{1 / 2}$ & $(5 / 2,1 / 2)_{3}$ & $2792.580^{\mathrm{B}}$ & $2795.105^{a}$ & $\begin{array}{l}-1.576^{\mathrm{A}} \\
-1.640^{\mathrm{B}}\end{array}$ & $\begin{array}{l}-1.32^{a 1} \\
-1.69^{a 2}\end{array}$ & $\begin{array}{l}2.20 \times 10^{7 A} \\
1.96 \times 10^{7 B}\end{array}$ & - \\
\hline $4 f 5 d$ & ${ }^{3} G_{3}^{o}$ & $5 d^{2}$ & ${ }^{3} F_{3}$ & $2796.770^{\mathrm{B}}$ & $2802.389^{a}$ & $-1.063^{\mathrm{A}}$ & $\begin{array}{l}-1.45^{a 1} \\
-1.25^{a 2}\end{array}$ & $\begin{array}{l}7.46 \times 10^{7 A} \\
9.16 \times 10^{7 B}\end{array}$ & \\
\hline $4 f 5 d$ & ${ }^{3} G_{5}^{o}$ & $5 d^{2}$ & ${ }^{3} F_{4}$ & $2937.511^{\mathrm{B}}$ & $2923.809^{a}$ & $-0.386^{\mathrm{A}}$ & $\begin{array}{c}-0.55^{a 1} \\
-0.33^{a 2, b}\end{array} \mid$ & $3.49 \times 10^{8 B}$ & $3.608 \times 10^{8 b}$ \\
\hline
\end{tabular}


TABLE I (cont.)

Wavelengths, $\lambda$, logarithmic weighted oscillator strengths, $\log (g f)$, and weighted transition probabilities, $g A_{k i}$, for electric dipole (E1) transitions in Ce III.

\begin{tabular}{|c|c|c|c|c|c|c|c|c|c|}
\hline \multicolumn{4}{|c|}{ Transition } & \multicolumn{2}{|c|}{$\bar{\lambda} \lambda[\AA]$} & \multicolumn{2}{|c|}{$\log (g f)$} & \multicolumn{2}{|c|}{$g A_{k i}\left[\mathrm{~s}^{-1}\right]$} \\
\hline \multicolumn{2}{|c|}{ Lower Level } & \multicolumn{2}{|c|}{ Upper level } & This & Other & This & Other & This & Other \\
\hline $4 f 5 d$ & ${ }^{3} G_{3}^{o}$ & $5 d^{2}$ & ${ }^{3} F_{2}$ & $2924.589^{\mathrm{A}}$ & $2925.260^{a}$ & $-0.712^{\mathrm{B}}$ & $\begin{array}{c}-0.83^{a 1} \\
-0.62^{a 2, b}\end{array}$ & $1.64 \times 10^{8 A}$ & $1.889 \times 10^{8 b}$ \\
\hline $4 f 5 d$ & ${ }^{3} G_{4}^{o}$ & $5 d^{2}$ & ${ }^{3} F_{3}$ & $2939.521^{\mathrm{B}}$ & $\begin{array}{l}2931.537^{a} \\
2931.538^{b}\end{array}$ & $-0.704^{\mathrm{B}}$ & $\begin{array}{l}-0.73^{a 1} \\
-0.51^{a 2, b}\end{array}$ & $1.68 \times 10^{8 A}$ & $2.398 \times 10^{8 b}$ \\
\hline $4 f 5 d$ & ${ }^{1} H_{5}^{o}$ & $4 f\left({ }^{2} F_{7 / 2}^{o}\right) 6 p_{1 / 2}$ & $(7 / 2,1 / 2)_{4}$ & $2879.217^{\mathrm{B}}$ & $\begin{array}{l}2948.534^{b} \\
2948.531^{c}\end{array}$ & $-1.237^{\mathrm{A}}$ & $\begin{array}{l}-2.17^{b} \\
-1.88^{c}\end{array}$ & $5.18 \times 10^{7 A}$ & $\begin{array}{c}5.212 \times 10^{6 b} \\
1.18 \times 10^{7 c}\end{array}$ \\
\hline $4 f 5 d$ & ${ }^{3} D_{3}^{o}$ & $5 d^{2}$ & ${ }^{3} F_{4}$ & $3009.837^{\mathrm{B}}$ & $2993.951^{b}$ & $-2.162^{\mathrm{A}}$ & $-2.11^{b}$ & $5.19 \times 10^{6 A}$ & $5.803 \times 10^{6 b}$ \\
\hline $4 f\left({ }^{2} F_{7 / 2}^{o}\right) 6 s_{1 / 2}$ & $(7 / 2,1 / 2)_{4}^{o}$ & $4 f\left({ }^{2} F_{7 / 2}^{o}\right) 6 p_{3 / 2}$ & $(7 / 2,3 / 2)_{4}$ & $3027.501^{\mathrm{B}}$ & $\mid \begin{array}{c}3022.745^{a, c} \\
3022.747^{b}\end{array}$ & $-0.335^{\mathrm{B}}$ & $\begin{array}{c}-0.22^{a 1} \\
-0.18^{a 2, c} \\
-0.10^{a 3, b} \\
-0.182^{d}\end{array}$ & $3.37 \times 10^{8 B}$ & $\begin{array}{c}5.788 \times 10^{8 b} \\
5.70 \times 10^{8 c}\end{array}$ \\
\hline $4 f\left({ }^{2} F_{7 / 2}^{o}\right) 6 s_{1 / 2}$ & $(7 / 2,1 / 2)_{4}^{o}$ & $4 f\left({ }^{2} F_{7 / 2}^{o}\right) 6 p_{3 / 2}$ & $(7 / 2,3 / 2)_{5}$ & $3091.707^{\mathrm{A}}$ & $\mid \begin{array}{c}3055.591^{a, c} \\
3055.589^{b}\end{array}$ & $\begin{array}{l}0.726^{\mathrm{A}} \\
0.714^{\mathrm{B}}\end{array}$ & $\begin{array}{c}0.63^{a 1} \\
0.74^{a 2} \\
0.73^{a 3, b}\end{array}$ & $3.71 \times 10^{9 A}$ & $3.818 \times 10^{9 b}$ \\
\hline $4 f\left({ }^{2} F_{7 / 2}^{o}\right) 6 s_{1 / 2}$ & $(7 / 2,1 / 2)_{3}^{o}$ & $4 f\left({ }^{2} F_{7 / 2}^{o}\right) 6 p_{3 / 2}$ & $(7 / 2,3 / 2)_{2}$ & $3012.804^{\mathrm{A}}$ & $\begin{array}{l}3056.560^{a} \\
3056.562^{b}\end{array}$ & $0.171^{\mathrm{A}}$ & $\begin{array}{c}0.21^{a 1} \\
0.28^{a 2} \\
0.30^{a 3, b} \\
0.278^{d}\end{array}$ & $1.09 \times 10^{9 A}$ & $1.431 \times 10^{9 b}$ \\
\hline $4 f\left({ }^{2} F_{7 / 2}^{o}\right) 6 s_{1 / 2}$ & $(7 / 2,1 / 2)_{3}^{o}$ & $4 f\left({ }^{2} F_{7 / 2}^{o}\right) 6 p_{3 / 2}$ & $(7 / 2,3 / 2)_{4}$ & $3061.529^{\mathrm{B}}$ & $\mid \begin{array}{c}3057.227^{a, c} \\
3057.229^{b}\end{array}$ & $0.214^{\mathrm{A}}$ & $\begin{array}{c}0.30^{a 1} \\
0.44^{a 2} \\
0.40^{a 3, b} \\
0.33^{a 4, c}\end{array}$ & $1.14 \times 10^{9 A}$ & $\begin{array}{c}1.787 \times 10^{9 b} \\
1.80 \times 10^{9 c}\end{array}$ \\
\hline $4 f 5 d$ & ${ }^{3} D_{2}^{o}$ & $5 d^{2}$ & ${ }^{3} F_{3}$ & $3118.562^{\mathrm{A}}$ & $3120.383^{b}$ & $-2.370^{\mathrm{B}}$ & $-2.19^{b}$ & $3.83 \times 10^{6 A}$ & $4.396 \times 10^{6 b}$ \\
\hline $4 f\left({ }^{2} F_{5 / 2}^{o}\right) 6 s_{1 / 2}$ & $(5 / 2,1 / 2)_{2}^{O}$ & $4 f\left({ }^{2} F_{5 / 2}^{o}\right) 6 p_{3 / 2}$ & $(5 / 2,3 / 2)_{3}$ & $3101.919^{\mathrm{A}}$ & $\mid \begin{array}{c}3121.560^{a, c} \\
3121.559^{b}\end{array}$ & $0.261^{\mathrm{A}}$ & $\begin{array}{c}0.19^{a 1} \\
0.31^{a 2, b} \\
0.20^{a 3, c} \\
0.311^{d}\end{array}$ & $1.26 \times 10^{9 A}$ & $\begin{array}{c}1.383 \times 10^{9 b} \\
1.35 \times 10^{9 c}\end{array}$ \\
\hline $4 f\left({ }^{2} F_{7 / 2}^{o}\right) 6 s_{1 / 2}$ & $(7 / 2,1 / 2)_{3}^{o}$ & $4 f\left({ }^{2} F_{7 / 2}^{o}\right) 6 p_{3 / 2}$ & $(7 / 2,3 / 2)_{3}$ & $3110.320^{\mathrm{B}}$ & $\mid \begin{array}{c}3147.058^{a, c} \\
3147.062^{b}\end{array}$ & $0.328^{\mathrm{A}}$ & $\begin{array}{c}0.14^{a 1} \\
0.20^{a 2} \\
0.23^{a 3, b} \\
0.22^{a 4, c} \\
0.201^{d}\end{array}$ & $1.38 \times 10^{9 A}$ & $\begin{array}{c}1.149 \times 10^{9 b} \\
1.30 \times 10^{9 c}\end{array}$ \\
\hline $4 f 5 d$ & ${ }^{3} D_{1}^{o}$ & $5 d^{2}$ & ${ }^{3} F_{2}$ & $3177.816^{\mathrm{B}}$ & $3171.848^{b}$ & $-2.342^{\mathrm{B}}$ & $-2.28^{b}$ & $3.01 \times 10^{6 B}$ & $3.459 \times 10^{6 b}$ \\
\hline $4 f\left({ }^{2} F_{5 / 2}^{o}\right) 6 s_{1 / 2}$ & $(5 / 2,1 / 2)_{2}^{O}$ & $5 d^{2}$ & ${ }^{3} P_{2}$ & $3246.110^{\mathrm{B}}$ & $3245.009^{b}$ & $-2.216^{\mathrm{A}}$ & $-1.48^{b}$ & $4.25 \times 10^{6 A}$ & $2.089 \times 10^{7 b}$ \\
\hline $4 f\left({ }^{2} F_{7 / 2}^{o}\right) 6 s_{1 / 2}$ & $(7 / 2,1 / 2)_{3}^{o}$ & $4 f\left({ }^{2} F_{5 / 2}^{o}\right) 6 p_{3 / 2}$ & $(5 / 2,3 / 2)_{4}$ & $3143.680^{\mathrm{B}}$ & $\mid \begin{array}{c}3267.941^{a, c} \\
3267.937^{b}\end{array}$ & $-0.775^{\mathrm{A}}$ & $\begin{array}{c}-0.79^{a 1} \\
-0.76^{a 2} \\
-0.73^{a 3, b} \\
-0.83^{a 4, c} \\
-0.761^{d}\end{array}$ & $0.93 \times 10^{8 A}$ & $\begin{array}{c}1.159 \times 10^{8 b} \\
1.15 \times 10^{8 c}\end{array}$ \\
\hline $4 f\left({ }^{2} F_{7 / 2}^{o}\right) 6 s_{1 / 2}$ & $(7 / 2,1 / 2)_{4}^{o}$ & $4 f\left({ }^{2} F_{7 / 2}^{o}\right) 6 p_{1 / 2}$ & $(7 / 2,1 / 2)_{4}$ & $3386.871^{\mathrm{B}}$ & $\mid \begin{array}{c}3497.810^{a, c} \\
3497.813^{b}\end{array}$ & $-0.500^{\mathrm{A}}$ & $\begin{array}{c}-0.39^{a 1} \\
-0.69^{a 2} \\
-0.31^{a 3, b} \\
-0.35^{a 4, c} \\
-0.689^{d}\end{array}$ & $2.03 \times 10^{8 A}$ & $\begin{array}{c}2.672 \times 10^{8 b} \\
2.80 \times 10^{8 c}\end{array}$ \\
\hline $4 f 5 d$ & ${ }^{1} P_{1}^{o}$ & $5 d^{2}$ & ${ }^{1} D_{2}$ & $3506.372^{\mathrm{B}}$ & $3514.409^{b}$ & $-1.496^{\mathrm{B}}$ & $-1.47^{b}$ & $1.99 \times 10^{7 A}$ & $1.825 \times 10^{7 b}$ \\
\hline $4 f\left({ }^{2} F_{5 / 2}^{o}\right) 6 s_{1 / 2}$ & $(5 / 2,1 / 2)_{3}^{o}$ & $5 d^{2}$ & ${ }^{1} D_{2}$ & $3645.337^{\mathrm{B}}$ & $3645.224^{b}$ & $-1.391^{\mathrm{A}}$ & $-1.36^{b}$ & $1.96 \times 10^{7 A}$ & $2.169 \times 10^{7 b}$ \\
\hline $4 f\left({ }^{2} F_{7 / 2}^{o}\right) 6 s_{1 / 2}$ & $(7 / 2,1 / 2)_{3}^{o}$ & $4 f\left({ }^{2} F_{5 / 2}^{o}\right) 6 p_{1 / 2}$ & $(5 / 2,1 / 2)_{3}$ & $3750.804^{\mathrm{B}}$ & $\mid \begin{array}{c}3784.290^{a, c} \\
3784.288^{b}\end{array}$ & $-1.006^{\mathrm{A}}$ & $\begin{array}{l}-1.21^{a 1} \\
-1.01^{a 2, b} \\
-1.04^{a 3, c}\end{array}$ & $4.47 \times 10^{7 A}$ & $\begin{array}{c}4.543 \times 10^{7 b} \\
5.09 \times 10^{7 c}\end{array}$ \\
\hline $4 f\left({ }^{2} F_{5 / 2}^{o}\right) 6 s_{1 / 2}$ & $(5 / 2,1 / 2)_{3}^{o}$ & $5 d^{2}$ & ${ }^{3} F_{4}$ & $4187.116^{\mathrm{B}}$ & $4156.309^{b}$ & $-2.440^{\mathrm{B}}$ & $-2.45^{b}$ & $1.38 \times 10^{6 B}$ & $1.360 \times 10^{6 b}$ \\
\hline $4 f\left({ }^{2} F_{5 / 2}^{o}\right) 6 s_{1 / 2}$ & $(5 / 2,1 / 2)_{3}^{o}$ & $5 d^{2}$ & ${ }^{3} F_{3}$ & $4434.919^{\mathrm{A}}$ & $4448.323^{b}$ & $-1.852^{\mathrm{B}}$ & $-1.81^{b}$ & $4.68 \times 10^{6 B}$ & $5.235 \times 10^{6 b}$ \\
\hline $4 f\left({ }^{2} F_{7 / 2}^{o}\right) 6 s_{1 / 2}$ & $(7 / 2,1 / 2)_{4}^{o}$ & $5 d^{2}$ & ${ }^{3} F_{4}$ & $4570.446^{\mathrm{B}}$ & $4535.726^{b}$ & $-1.359^{\mathrm{A}}$ & $-1.51^{b}$ & $1.45 \times 10^{7 A}$ & $10.04 \times 10^{7 b}$ \\
\hline
\end{tabular}


TABLE I cont.

Wavelengths, $\lambda$, logarithmic weighted oscillator strengths, $\log (g f)$, and weighted transition probabilities, $g A_{k i}$, for electric dipole (E1) transitions in Ce III.

\begin{tabular}{|c|c|c|c|c|c|c|c|c|c|}
\hline \multicolumn{4}{|c|}{ Transition } & \multicolumn{2}{|c|}{$\lambda[\AA]$} & \multicolumn{2}{|c|}{$\log (g f)$} & \multicolumn{2}{|c|}{$g A_{k i}\left[\mathrm{~s}^{-1}\right]$} \\
\hline \multicolumn{2}{|c|}{ Lower Level } & \multicolumn{2}{|c|}{ Upper level } & This & Other & This & Other & This & Other \\
\hline $4 f\left({ }^{2} F_{7 / 2}^{o}\right) 6 s_{1 / 2}$ & $(7 / 2,1 / 2)_{3}^{o}$ & $5 d^{2}$ & ${ }^{3} F_{4}$ & $4648.444^{\mathrm{B}}$ & $4613.803^{b}$ & $-2.573^{\mathrm{B}}$ & $-2.96^{b}$ & $8.25 \times 10^{5 B}$ & $3.409 \times 10^{5 b}$ \\
\hline $4 f 5 d$ & ${ }^{3} P_{1}^{o}$ & $4 f^{2}$ & ${ }^{1} S_{0}$ & $4694.083^{\mathrm{B}}$ & $4709.904^{b}$ & $-3.702^{\mathrm{B}}$ & $-3.70^{b}$ & $6.01 \times 10^{4 B}$ & $5.390 \times 10^{4 b}$ \\
\hline $4 f\left({ }^{2} F_{5 / 2}^{o}\right) 6 s_{1 / 2}$ & $(5 / 2,1 / 2)_{3}^{o}$ & $5 d^{2}$ & ${ }^{3} F_{2}$ & $4784.215^{\mathrm{B}}$ & $4766.071^{b}$ & $-2.625^{\mathrm{B}}$ & $-2.57^{b}$ & $6.91 \times 10^{5 B}$ & $7.904 \times 10^{5 b}$ \\
\hline $4 f\left({ }^{2} F_{7 / 2}^{o}\right) 6 s_{1 / 2}$ & $(7 / 2,1 / 2)_{4}^{o}$ & $5 d^{2}$ & ${ }^{3} F_{3}$ & $4869.911^{\mathrm{A}}$ & $4885.730^{b}$ & $-2.391^{\mathrm{A}}$ & $-2.59^{b}$ & $11.4 \times 10^{5 A}$ & $7.224 \times 10^{5 b}$ \\
\hline $4 f\left({ }^{2} F_{7 / 2}^{o}\right) 6 s_{1 / 2}$ & $(7 / 2,1 / 2)_{3}^{o}$ & $5 d^{2}$ & ${ }^{3} F_{3}$ & $4961.008^{\mathrm{A}}$ & $4976.447^{b}$ & $-2.256^{\mathrm{A}}$ & $-2.37^{b}$ & $1.50 \times 10^{6 A}$ & $1.160 \times 10^{6 b}$ \\
\hline $4 f^{2}$ & ${ }^{3} F_{2}$ & $4 f\left({ }^{2} F_{5 / 2}^{o}\right) 6 s_{1 / 2}$ & $(5 / 2,1 / 2)_{2}^{o}$ & $6461.343^{\mathrm{B}}$ & $6460.866^{b}$ & $-3.161^{\mathrm{B}}$ & $-3.52^{b}$ & $1.10 \times 10^{5 B}$ & $4.278 \times 10^{4 b}$ \\
\hline $4 f 5 d$ & ${ }^{1} P_{1}^{o}$ & $4 f^{2}$ & ${ }^{1} S_{0}$ & $6961.863^{\mathrm{A}}$ & $6944.935^{b}$ & $-1.653^{\mathrm{B}}$ & $-1.63^{b}$ & $3.15 \times 10^{6 B}$ & $3.253 \times 10^{6 b}$ \\
\hline $4 f^{2}$ & ${ }^{3} \mathrm{H}_{6}$ & $4 f 5 d$ & ${ }^{1} H_{5}^{o}$ & $7660.727^{\mathrm{B}}$ & $7675.272^{b}$ & $-2.847^{\mathrm{A}}$ & $-2.89^{b}$ & $1.60 \times 10^{5 A}$ & $1.468 \times 10^{5 b}$ \\
\hline $4 f^{2}$ & ${ }^{3} \mathrm{H}_{4}$ & $4 f 5 d$ & ${ }^{1} F_{3}^{o}$ & $7920.536^{\mathrm{B}}$ & $7997.318^{b}$ & $-3.469^{\mathrm{B}}$ & $-3.40^{b}$ & $3.61 \times 10^{4 B}$ & $4.033 \times 10^{4 b}$ \\
\hline $4 f^{2}$ & ${ }^{3} F_{4}$ & $4 f 5 d$ & ${ }^{1} H_{5}^{o}$ & $8929.883^{\mathrm{B}}$ & $8969.177^{b}$ & $-3.252^{\mathrm{A}}$ & $-3.30^{b}$ & $4.59 \times 10^{4 A}$ & $4.511 \times 10^{4 b}$ \\
\hline $4 f 5 d$ & ${ }^{3} H_{5}^{o}$ & $4 f^{2}$ & ${ }^{1} I_{6}$ & $8752.491^{\mathrm{B}}$ & $9039.667^{b}$ & $-3.417^{\mathrm{A}}$ & $-3.52^{b}$ & $3.36 \times 10^{4 A}$ & $2.454 \times 10^{4 b}$ \\
\hline
\end{tabular}

$\overline{a, a 1, a 2, a 3, a 4}$ Ref. [1]; ${ }^{b}$ Ref. [6]; ${ }^{c}$ Ref. [7]; ${ }^{d}$ Ref. [12].

TABLE II

$\lambda, \log (g f)$, and $g A_{k i}$ for electric dipole (E1) transitions in Ce III. The lower and upper levels of each transition are presented by their $J$-values, parities [(e) for even and (o) for odd], and energy calculation results [ $\left.E_{\mathrm{th}}, 16\right]$ and experimental values [ $\left.E_{\text {exp }}, 23\right]$.

\begin{tabular}{|c|c|c|c|c|c|c|c|c|c|c|}
\hline \multicolumn{8}{|c|}{ Transition } & \multirow{3}{*}{$\lambda[\AA]$} & \multirow{3}{*}{$\log (g f)$} & \multirow{3}{*}{$g A_{k i}\left[\mathrm{~s}^{-1}\right]$} \\
\hline \multicolumn{4}{|c|}{ Lower level } & \multicolumn{4}{|c|}{ Upper level } & & & \\
\hline$J$ & Parity & $E_{\mathrm{th}}\left[\mathrm{cm}^{-1}\right]$ & $E_{\exp }\left[\mathrm{cm}^{-1}\right]$ & $J$ & Parity & $E_{\mathrm{th}}\left[\mathrm{cm}^{-1}\right]$ & $E_{\exp }\left[\mathrm{cm}^{-1}\right]$ & & & \\
\hline \multirow[t]{2}{*}{6} & (o) & $8159.59^{\mathrm{B}}$ & 8349.99 & 5 & (e) & $101666.49^{\mathrm{A}}$ & 101178.46 & $1069.175^{\mathrm{A}}$ & $-1.472^{\mathrm{A}}$ & $1.97 \times 10^{8 A}$ \\
\hline & & & & & & & & $1065.353^{\mathrm{B}}$ & $-1.163^{\mathrm{B}}$ & $4.04 \times 10^{8 B}$ \\
\hline \multirow[t]{2}{*}{4} & (e) & $0.00^{\mathrm{B}}$ & 0.00 & 3 & (o) & $92670.48^{\mathrm{A}}$ & 92705.16 & $1079.092^{\mathrm{A}}$ & $-3.445^{\mathrm{A}}$ & $2.06 \times 10^{6 A}$ \\
\hline & & & & & & & & $1093.698^{\mathrm{B}}$ & $-3.915^{\mathrm{B}}$ & $6.78 \times 10^{5 B}$ \\
\hline \multirow[t]{2}{*}{6} & (o) & $8159.59^{\mathrm{B}}$ & 8349.99 & 6 & (e) & $99924.94^{\mathrm{A}}$ & 100015.70 & $1089.461^{\mathrm{A}}$ & $-1.701^{\mathrm{A}}$ & $1.12 \times 10^{8 A}$ \\
\hline & & & & & & & & $1086.428^{\mathrm{B}}$ & $-1.421^{\mathrm{B}}$ & $2.15 \times 10^{8 B}$ \\
\hline \multirow[t]{2}{*}{6} & (o) & $8159.59^{\mathrm{B}}$ & 8349.99 & 5 & (e) & $99593.06^{\mathrm{A}}$ & 99604.30 & $1093.414^{\mathrm{A}}$ & $-2.344^{\mathrm{A}}$ & $2.53 \times 10^{7 A}$ \\
\hline & & & & & & & & $1088.291^{\mathrm{B}}$ & $-2.721^{\mathrm{B}}$ & $1.07 \times 10^{7 B}$ \\
\hline \multirow[t]{2}{*}{5} & (e) & $1535.39^{\mathrm{B}}$ & 1528.32 & 4 & (o) & $91705.38^{\mathrm{A}}$ & 91735.87 & $1108.750^{\mathrm{A}}$ & $-2.025^{\mathrm{A}}$ & $5.12 \times 10^{7 A}$ \\
\hline & & & & & & & & $1107.260^{\mathrm{B}}$ & $-2.416^{\mathrm{B}}$ & $2.09 \times 10^{7 B}$ \\
\hline \multirow[t]{2}{*}{2} & (e) & $3696.42^{\mathrm{B}}$ & 3762.75 & 1 & (o) & $93734.68^{\mathrm{A}}$ & 93602.83 & $1110.194^{\mathrm{A}}$ & $-3.958^{\mathrm{A}}$ & $5.96 \times 10^{5 A}$ \\
\hline & & & & & & & & $1114.414^{\mathrm{B}}$ & $-3.154^{\mathrm{B}}$ & $3.76 \times 10^{6 B}$ \\
\hline \multirow[t]{2}{*}{4} & (e) & $0.00^{\mathrm{B}}$ & 0.00 & 3 & (o) & $89698.61^{\mathrm{A}}$ & 89743.68 & $1114.844^{\mathrm{A}}$ & $-1.951^{\mathrm{A}}$ & $6.01 \times 10^{7 A}$ \\
\hline & & & & & & & & $1110.044^{\mathrm{B}}$ & $-2.023^{\mathrm{B}}$ & $5.14 \times 10^{7 B}$ \\
\hline \multirow[t]{2}{*}{6} & (e) & $3147.01^{\mathrm{B}}$ & 3127.10 & 6 & (o) & $92422.91^{\mathrm{A}}$ & 92526.56 & $1119.627^{\mathrm{A}}$ & $-2.488^{\mathrm{A}}$ & $1.73 \times 10^{7 A}$ \\
\hline & & & & & & & & $1122.264^{\mathrm{B}}$ & $-2.453^{\mathrm{B}}$ & $1.87 \times 10^{7 B}$ \\
\hline \multirow[t]{2}{*}{5} & (e) & $1535.39^{\mathrm{B}}$ & 1528.32 & 5 & (o) & $90544.71^{\mathrm{A}}$ & 90658.94 & $1123.204^{\mathrm{A}}$ & $-2.821^{\mathrm{A}}$ & $7.98 \times 10^{6 A}$ \\
\hline & & & & & & & & $1120.333^{\mathrm{B}}$ & $-2.792^{\mathrm{B}}$ & $8.58 \times 10^{6 B}$ \\
\hline \multirow[t]{2}{*}{5} & (e) & $1535.39^{\mathrm{B}}$ & 1528.32 & 4 & (o) & $90044.32^{\mathrm{A}}$ & 90045.27 & $1129.552^{\mathrm{A}}$ & $-2.140^{\mathrm{A}}$ & $3.79 \times 10^{7 A}$ \\
\hline & & & & & & & & $1130.751^{\mathrm{B}}$ & $-2.515^{\mathrm{B}}$ & $1.59 \times 10^{7 B}$ \\
\hline \multirow[t]{2}{*}{4} & (e) & $5002.26^{\mathrm{B}}$ & 5006.06 & 5 & (o) & $93431.88^{\mathrm{B}}$ & 93226.80 & $1136.162^{\mathrm{A}}$ & $-3.129^{\mathrm{A}}$ & $3.84 \times 10^{6 A}$ \\
\hline & & & & & & & & $1130.842^{\mathrm{B}}$ & $-2.708^{\mathrm{B}}$ & $1.02 \times 10^{7 B}$ \\
\hline \multirow[t]{2}{*}{5} & (e) & $1535.39^{\mathrm{B}}$ & 1528.32 & 4 & (o) & $89493.00^{\mathrm{A}}$ & 89651.91 & $1136.631^{\mathrm{A}}$ & $-3.620^{\mathrm{A}}$ & $1.24 \times 10^{6 A}$ \\
\hline & & & & & & & & $1127.628^{\mathrm{B}}$ & $-2.383^{\mathrm{B}}$ & $2.17 \times 10^{7 B}$ \\
\hline \multirow[t]{2}{*}{6} & (e) & $3147.01^{\mathrm{B}}$ & 3127.10 & 5 & (o) & $90544.71^{\mathrm{A}}$ & 90658.94 & $1143.677^{\mathrm{A}}$ & $-2.761^{\mathrm{A}}$ & $8.84 \times 10^{6 A}$ \\
\hline & & & & & & & & $1140.933^{\mathrm{B}}$ & $-2.608^{\mathrm{B}}$ & $1.26 \times 10^{7 B}$ \\
\hline \multirow[t]{2}{*}{3} & (e) & $4718.90^{\mathrm{B}}$ & 4764.76 & 4 & (o) & $92156.59^{\mathrm{A}}$ & 92080.62 & $1143.048^{\mathrm{A}}$ & $-3.736^{\mathrm{A}}$ & $9.38 \times 10^{5 A}$ \\
\hline & & & & & & & & $1156.997^{\mathrm{B}}$ & $-3.199^{\mathrm{B}}$ & $3.15 \times 10^{6 B}$ \\
\hline \multirow[t]{2}{*}{4} & (e) & $5002.26^{\mathrm{B}}$ & 5006.06 & 5 & (o) & $92189.92^{\mathrm{A}}$ & 92180.41 & $1146.940^{\mathrm{A}}$ & $-3.087^{\mathrm{A}}$ & $4.15 \times 10^{6 A}$ \\
\hline & & & & & & & & $1148.825^{\mathrm{B}}$ & $-3.270^{\mathrm{B}}$ & $2.72 \times 10^{6 B}$ \\
\hline
\end{tabular}


TABLE II (cont.)

$\lambda, \log (g f)$, and $g A_{k i}$ for electric dipole (E1) transitions in Ce III. The lower and upper levels of each transition are presented by their $J$-values, parities [(e) for even and (o) for odd], and energy calculation results [ $\left.E_{\mathrm{th}}, 16\right]$ and experimental values [ $\left.E_{\mathrm{exp}}, 23\right]$.

\begin{tabular}{|c|c|c|c|c|c|c|c|c|c|c|}
\hline \multicolumn{8}{|c|}{ Transition } & \multirow{3}{*}{$\lambda[\AA]$} & \multirow{3}{*}{$\log (g f)$} & \multirow{3}{*}{$g A_{k i}\left[\mathrm{~s}^{-1}\right]$} \\
\hline \multicolumn{4}{|c|}{ Lower level } & \multicolumn{4}{|c|}{ Upper level } & & & \\
\hline$J$ & Parity & $E_{\mathrm{th}}\left[\mathrm{cm}^{-1}\right]$ & $E_{\exp }\left[\mathrm{cm}^{-1}\right]$ & $J$ & Parity & $E_{\mathrm{th}}\left[\mathrm{cm}^{-1}\right]$ & $E_{\exp }\left[\mathrm{cm}^{-1}\right]$ & & & \\
\hline \multirow[t]{2}{*}{4} & (e) & $5002.26^{\mathrm{B}}$ & 5006.06 & 4 & (o) & $91705.38^{\mathrm{A}}$ & 91735.87 & $1153.350^{\mathrm{A}}$ & $-2.769^{\mathrm{A}}$ & $8.53 \times 10^{6 \mathrm{~A}}$ \\
\hline & & & & & & & & $1151.461^{\mathrm{B}}$ & $-3.302^{\mathrm{B}}$ & $2.51 \times 10^{6 B}$ \\
\hline \multirow[t]{2}{*}{2} & (e) & $3696.42^{\mathrm{B}}$ & 3762.75 & 1 & (o) & $90132.70^{\mathrm{B}}$ & 90144.52 & $1155.936^{\mathrm{A}}$ & $-3.398^{\mathrm{A}}$ & $2.00 \times 10^{6 \mathrm{~A}}$ \\
\hline & & & & & & & & $1156.921^{\mathrm{B}}$ & $-2.791^{\mathrm{B}}$ & $8.06 \times 10^{6 B}$ \\
\hline \multirow[t]{2}{*}{2} & (e) & $3696.42^{\mathrm{B}}$ & 3762.75 & 3 & (o) & $89698.61^{\mathrm{A}}$ & 89743.68 & $1162.274^{\mathrm{A}}$ & $-3.548^{\mathrm{A}}$ & $2.00 \times 10^{6 A}$ \\
\hline & & & & & & & & $1157.540^{\mathrm{B}}$ & $-3.469^{\mathrm{B}}$ & $1.69 \times 10^{6 B}$ \\
\hline \multirow[t]{2}{*}{4} & (e) & $5002.26^{\mathrm{B}}$ & 5006.06 & 5 & (o) & $90544.71^{\mathrm{A}}$ & 90658.94 & $1168.998^{\mathrm{A}}$ & $-3.406^{\mathrm{A}}$ & $1.92 \times 10^{6 A}$ \\
\hline & & & & & & & & $1165.606^{\mathrm{B}}$ & $-3.348^{\mathrm{B}}$ & $2.20 \times 10^{6 B}$ \\
\hline \multirow[t]{2}{*}{3} & (e) & $4718.90^{\mathrm{B}}$ & 4764.76 & 3 & (o) & $89917.29^{\mathrm{B}}$ & 90086.92 & $1169.984^{\mathrm{A}}$ & $-3.005^{\mathrm{A}}$ & $4.82 \times 10^{6 A}$ \\
\hline & & & & & & & & $1177.648^{\mathrm{B}}$ & $-3.442^{\mathrm{B}}$ & $1.74 \times 10^{6 B}$ \\
\hline \multirow[t]{2}{*}{3} & (e) & $4718.90^{\mathrm{B}}$ & 4764.76 & 4 & (o) & $90044.32^{\mathrm{A}}$ & 90045.27 & $1171.329^{\mathrm{A}}$ & $-3.656^{\mathrm{A}}$ & $1.07 \times 10^{6 A}$ \\
\hline & & & & & & & & $1176.887^{\mathrm{B}}$ & $-2.956^{\mathrm{B}}$ & $5.33 \times 10^{6 B}$ \\
\hline \multirow[t]{2}{*}{4} & (e) & $7118.91^{\mathrm{B}}$ & 7120.00 & 4 & (o) & $92156.59^{\mathrm{A}}$ & 92080.62 & $1176.116^{\mathrm{A}}$ & $-2.593^{\mathrm{A}}$ & $1.23 \times 10^{7 A}$ \\
\hline & & & & & & & & $1186.043^{\mathrm{B}}$ & $-3.009^{\mathrm{B}}$ & $4.64 \times 10^{6 B}$ \\
\hline \multirow[t]{2}{*}{4} & (e) & $7118.91^{\mathrm{B}}$ & 7120.00 & 5 & (o) & $90544.71^{\mathrm{A}}$ & 90658.94 & $1198.843^{\mathrm{A}}$ & $-3.406^{\mathrm{A}}$ & $1.82 \times 10^{6 A}$ \\
\hline & & & & & & & & $1195.091^{\mathrm{B}}$ & $-3.593^{\mathrm{B}}$ & $1.19 \times 10^{6 B}$ \\
\hline \multirow[t]{2}{*}{2} & (e) & $12844.70^{\mathrm{A}}$ & 12835.09 & 1 & (o) & $90865.83^{\mathrm{A}}$ & 90878.78 & $1281.704^{\mathrm{A}}$ & $-3.103^{\mathrm{A}}$ & $3.20 \times 10^{6 A}$ \\
\hline & & & & & & & & $1281.193^{\mathrm{B}}$ & $-3.044^{\mathrm{B}}$ & $3.67 \times 10^{6 B}$ \\
\hline \multirow[t]{2}{*}{2} & (e) & $12844.70^{\mathrm{A}}$ & 12835.09 & 2 & (o) & $92190.88^{\mathrm{A}}$ & 90223.72 & $1260.300^{\mathrm{A}}$ & $-3.346^{\mathrm{A}}$ & $1.89 \times 10^{6 \mathrm{~A}}$ \\
\hline & & & & & & & & $1290.034^{\mathrm{B}}$ & $-1.997^{\mathrm{B}}$ & $4.03 \times 10^{7 B}$ \\
\hline 2 & (e) & $12844.70^{\mathrm{A}}$ & 12835.09 & 3 & (o) & $89917.29^{\mathrm{B}}$ & 90086.92 & $1293.698^{\mathrm{A}}$ & $-3.008^{\mathrm{A}}$ & $3.92 \times 10^{6 A}$ \\
\hline & & & & & & & & $1296.341^{\mathrm{B}}$ & $-3.293^{\mathrm{B}}$ & $2.02 \times 10^{6 B}$ \\
\hline 2 & (e) & $12844.70^{\mathrm{A}}$ & 12835.09 & 2 & (o) & $89534.96^{\mathrm{A}}$ & 89350.03 & $1303.946^{\mathrm{A}}$ & $-3.271^{\mathrm{A}}$ & $2.10 \times 10^{6 A}$ \\
\hline & & & & & & & & $1315.155^{\mathrm{B}}$ & $-2.146^{\mathrm{B}}$ & $2.75 \times 10^{7 B}$ \\
\hline 2 & (e) & $17372.82^{\mathrm{A}}$ & 17317.49 & 1 & (o) & $93734.68^{\mathrm{A}}$ & 93602.83 & $1309.553^{\mathrm{A}}$ & $-3.064^{\mathrm{A}}$ & $3.36 \times 10^{6 A}$ \\
\hline & & & & & & & & $1315.120^{\mathrm{B}}$ & $-3.010^{\mathrm{B}}$ & $3.77 \times 10^{6 B}$ \\
\hline 1 & (e) & $16520.61^{\mathrm{B}}$ & 16523.66 & 2 & (o) & $92838.71^{\mathrm{A}}$ & 92795.44 & $1309.865^{\mathrm{A}}$ & $-2.841^{\mathrm{A}}$ & $5.61 \times 10^{6 A}$ \\
\hline & & & & & & & & $1317.573^{\mathrm{B}}$ & $-2.643^{\mathrm{B}}$ & $8.74 \times 10^{6 B}$ \\
\hline 6 & (e) & $17450.52^{\mathrm{B}}$ & 17420.60 & 5 & (o) & $93431.88^{\mathrm{B}}$ & 93226.80 & $1324.935^{\mathrm{A}}$ & $-2.365^{\mathrm{A}}$ & $1.64 \times 10^{7 A}$ \\
\hline & & & & & & & & $1316.111^{\mathrm{B}}$ & $-2.639^{\mathrm{B}}$ & $8.85 \times 10^{6 B}$ \\
\hline 0 & (e) & $16029.38^{\mathrm{B}}$ & 16072.04 & 1 & (o) & $90865.83^{\mathrm{A}}$ & 90878.78 & $1335.747^{\mathrm{A}}$ & $-2.707^{\mathrm{A}}$ & $7.34 \times 10^{6 A}$ \\
\hline & & & & & & & & $1336.899^{\mathrm{B}}$ & $-2.902^{\mathrm{B}}$ & $4.67 \times 10^{6 B}$ \\
\hline 6 & (e) & $17450.52^{\mathrm{B}}$ & 17420.60 & 5 & (o) & $92189.92^{\mathrm{A}}$ & 92180.41 & $1339.615^{\mathrm{A}}$ & $-3.018^{\mathrm{A}}$ & $3.57 \times 10^{6 A}$ \\
\hline & & & & & & & & $1340.532^{\mathrm{B}}$ & $-4.102^{\mathrm{B}}$ & $2.94 \times 10^{5 B}$ \\
\hline 1 & (e) & $16520.61^{\mathrm{B}}$ & 16523.66 & 0 & (o) & $90862.07^{\mathrm{A}}$ & 90902.41 & $1344.680^{\mathrm{A}}$ & $-2.621^{\mathrm{A}}$ & $8.82 \times 10^{6 A}$ \\
\hline & & & & & & & & $1347.605^{\mathrm{B}}$ & $-2.426^{\mathrm{B}}$ & $1.38 \times 10^{7 B}$ \\
\hline 1 & (e) & $16520.61^{\mathrm{B}}$ & 16523.66 & 1 & (o) & $90865.83^{\mathrm{A}}$ & 90878.78 & $1344.612^{\mathrm{A}}$ & $-2.704^{\mathrm{A}}$ & $7.29 \times 10^{6 A}$ \\
\hline & & & & & & & & $1345.737^{\mathrm{B}}$ & $-2.363^{\mathrm{B}}$ & $1.59 \times 10^{7 B}$ \\
\hline 0 & (e) & $16029.38^{\mathrm{B}}$ & 16072.04 & 1 & (o) & $90132.70^{\mathrm{B}}$ & 90144.52 & $1348.271^{\mathrm{A}}$ & $-2.695^{\mathrm{A}}$ & $7.41 \times 10^{6 \mathrm{~A}}$ \\
\hline & & & & & & & & $1349.466^{\mathrm{B}}$ & $-2.007^{\mathrm{B}}$ & $3.60 \times 10^{7 B}$ \\
\hline 1 & (e) & $16520.61^{\mathrm{B}}$ & 16523.66 & 1 & (o) & $90132.70^{\mathrm{B}}$ & 90144.52 & $1357.304^{\mathrm{A}}$ & $-3.081^{\mathrm{A}}$ & $3.00 \times 10^{6 \mathrm{~A}}$ \\
\hline & & & & & & & & $1358.472^{\mathrm{B}}$ & $-3.299^{\mathrm{B}}$ & $1.82 \times 10^{6 B}$ \\
\hline 2 & (e) & $17372.82^{\mathrm{A}}$ & 17317.49 & 1 & (o) & $90865.83^{\mathrm{A}}$ & 90878.78 & $1360.673^{\mathrm{A}}$ & $-2.702^{\mathrm{A}}$ & $7.15 \times 10^{6 A}$ \\
\hline & & & & & & & & $1361.687^{\mathrm{B}}$ & $-2.466^{\mathrm{B}}$ & $1.23 \times 10^{7 B}$ \\
\hline 6 & (e) & $17450.52^{\mathrm{B}}$ & 17420.60 & 5 & (o) & $90544.71^{\mathrm{A}}$ & 90658.94 & $1369.805^{\mathrm{A}}$ & $-2.836^{\mathrm{A}}$ & $5.19 \times 10^{6 A}$ \\
\hline & & & & & & & & $1363.437^{\mathrm{B}}$ & $-3.340^{\mathrm{B}}$ & $1.64 \times 10^{6 B}$ \\
\hline 2 & (e) & $17372.82^{\mathrm{A}}$ & 17317.49 & 2 & (o) & $92190.88^{\mathrm{A}}$ & 90223.72 & $1336.575^{\mathrm{A}}$ & $-3.481^{\mathrm{A}}$ & $1.23 \times 10^{6 \mathrm{~A}}$ \\
\hline & & & & & & & & $1371.677^{\mathrm{B}}$ & $-2.692^{\mathrm{B}}$ & $7.20 \times 10^{6 B}$ \\
\hline 3 & (o) & $6035.12^{\mathrm{A}}$ & 6265.21 & 2 & (e) & $70452.40^{\mathrm{B}}$ & 70433.08 & $1551.266^{\mathrm{A}}$ & $-2.628^{\mathrm{A}}$ & $6.52 \times 10^{6 A}$ \\
\hline & & & & & & & & $1551.601^{\mathrm{B}}$ & $-2.899^{\mathrm{B}}$ & $3.50 \times 10^{6 B}$ \\
\hline 4 & (o) & $3372.91^{\mathrm{B}}$ & 3276.66 & 3 & (e) & $65587.20^{\mathrm{A}}$ & 65550.73 & $1608.865^{\mathrm{A}}$ & $-3.672^{\mathrm{A}}$ & $5.48 \times 10^{5 A}$ \\
\hline & & & & & & & & $1610.940^{\mathrm{B}}$ & $-4.164^{\mathrm{B}}$ & $1.76 \times 10^{5 B}$ \\
\hline
\end{tabular}


TABLE II (cont.)

$\lambda, \log (g f)$, and $g A_{k i}$ for electric dipole (E1) transitions in Ce III. The lower and upper levels of each transition are presented by their $J$-values, parities [(e) for even and (o) for odd], and energy calculation results [ $\left.E_{\mathrm{th}}, 16\right]$ and experimental values [ $\left.E_{\text {exp }}, 23\right]$.

\begin{tabular}{|c|c|c|c|c|c|c|c|c|c|c|}
\hline \multicolumn{8}{|c|}{ Transition } & \multirow{3}{*}{$\lambda[\AA]$} & \multirow{3}{*}{$\log (g f)$} & \multirow{3}{*}{$g A_{k i}\left[\mathrm{~s}^{-1}\right]$} \\
\hline \multicolumn{4}{|c|}{ Lower level } & \multicolumn{4}{|c|}{ Upper level } & & & \\
\hline$J$ & Parity & $E_{\mathrm{th}}\left[\mathrm{cm}^{-1}\right]$ & $E_{\exp }\left[\mathrm{cm}^{-1}\right]$ & $J$ & Parity & $E_{\mathrm{th}}\left[\mathrm{cm}^{-1}\right]$ & $E_{\exp }\left[\mathrm{cm}^{-1}\right]$ & & & \\
\hline \multirow[t]{2}{*}{0} & (e) & $32890.30^{\mathrm{B}}$ & 32838.62 & 1 & (o) & $93734.68^{\mathrm{A}}$ & 93602.83 & $1640.540^{\mathrm{A}}$ & $-1.516^{\mathrm{A}}$ & $7.55 \times 10^{7 A}$ \\
\hline & & & & & & & & $1651.818^{\mathrm{B}}$ & $-1.443^{\mathrm{B}}$ & $8.81 \times 10^{7 B}$ \\
\hline \multirow[t]{2}{*}{4} & (o) & $5020.45^{\mathrm{A}}$ & 5127.27 & 3 & (e) & $65587.20^{\mathrm{A}}$ & 65550.73 & $1651.070^{\mathrm{A}}$ & $-3.090^{\mathrm{A}}$ & $1.99 \times 10^{6 A}$ \\
\hline & & & & & & & & $1654.778^{\mathrm{B}}$ & $-3.197^{\mathrm{B}}$ & $1.55 \times 10^{6 B}$ \\
\hline \multirow[t]{2}{*}{3} & (o) & $10148.74^{\mathrm{A}}$ & 10126.53 & 2 & (e) & $70452.40^{\mathrm{B}}$ & 70433.08 & $1657.005^{\mathrm{A}}$ & $-1.510^{\mathrm{A}}$ & $7.52 \times 10^{7 A}$ \\
\hline & & & & & & & & $1655.886^{\mathrm{B}}$ & $-1.640^{\mathrm{B}}$ & $5.57 \times 10^{7 B}$ \\
\hline \multirow[t]{2}{*}{2} & (o) & $3843.05^{\mathrm{B}}$ & 3821.53 & 2 & (e) & $64022.28^{\mathrm{A}}$ & 64010.70 & $1664.672^{\mathrm{A}}$ & $-2.409^{\mathrm{A}}$ & $9.38 \times 10^{6 A}$ \\
\hline & & & & & & & & $1660.227^{\mathrm{B}}$ & $-2.707^{\mathrm{B}}$ & $4.75 \times 10^{6 B}$ \\
\hline \multirow[t]{2}{*}{2} & (o) & $3843.05^{\mathrm{B}}$ & 3821.53 & 1 & (e) & $63348.51^{\mathrm{A}}$ & 63335.40 & $1683.554^{\mathrm{A}}$ & $-2.091^{\mathrm{A}}$ & $1.91 \times 10^{7 A}$ \\
\hline & & & & & & & & $1676.283^{\mathrm{B}}$ & $-2.043^{\mathrm{B}}$ & $2.15 \times 10^{7 B}$ \\
\hline \multirow[t]{2}{*}{3} & (o) & $6035.12^{\mathrm{A}}$ & 6265.21 & 3 & (e) & $65587.20^{\mathrm{A}}$ & 65550.73 & $1679.202^{\mathrm{A}}$ & $-3.431^{\mathrm{A}}$ & $8.76 \times 10^{5 A}$ \\
\hline & & & & & & & & $1682.210^{\mathrm{B}}$ & $-3.487^{\mathrm{B}}$ & $7.68 \times 10^{5 B}$ \\
\hline \multirow[t]{2}{*}{1} & (o) & $8851.76^{\mathrm{A}}$ & 8922.05 & 0 & (e) & $67656.90^{\mathrm{A}}$ & 67730.30 & $1700.532^{\mathrm{A}}$ & $-2.685^{\mathrm{A}}$ & $4.77 \times 10^{6 A}$ \\
\hline & & & & & & & & $1700.405^{\mathrm{B}}$ & $-2.529^{\mathrm{B}}$ & $6.82 \times 10^{6 B}$ \\
\hline \multirow[t]{2}{*}{3} & (o) & $5479.13^{\mathrm{B}}$ & 5502.37 & 2 & (e) & $64022.28^{\mathrm{A}}$ & 64010.70 & $1709.906^{\mathrm{A}}$ & $-1.809^{\mathrm{A}}$ & $3.54 \times 10^{7 A}$ \\
\hline & & & & & & & & $1706.583^{\mathrm{B}}$ & $-1.874^{\mathrm{B}}$ & $3.06 \times 10^{7 B}$ \\
\hline \multirow[t]{2}{*}{4} & (o) & $7262.13^{\text {B }}$ & 7150.05 & 3 & (e) & $65587.20^{\mathrm{A}}$ & 65550.73 & $1714.985^{\mathrm{A}}$ & $-1.961^{\mathrm{A}}$ & $2.48 \times 10^{7 A}$ \\
\hline & & & & & & & & $1718.616^{\mathrm{B}}$ & $-2.136^{\mathrm{B}}$ & $1.65 \times 10^{7 B}$ \\
\hline \multirow[t]{2}{*}{0} & (e) & $32890.30^{\mathrm{B}}$ & 32838.62 & 1 & (o) & $90865.83^{\mathrm{A}}$ & 90878.78 & $1721.566^{\mathrm{A}}$ & $-2.251^{\mathrm{A}}$ & $1.26 \times 10^{7 A}$ \\
\hline & & & & & & & & $1725.953^{\mathrm{B}}$ & $-2.661^{\mathrm{B}}$ & $4.89 \times 10^{6 B}$ \\
\hline 3 & (o) & $12625.38^{\mathrm{B}}$ & 12500.72 & 2 & (e) & $70452.40^{\mathrm{B}}$ & 70433.08 & $1730.149^{\mathrm{A}}$ & $-0.846^{\mathrm{A}}$ & $3.18 \times 10^{8 A}$ \\
\hline & & & & & & & & $1729.295^{\mathrm{B}}$ & $-1.059^{\mathrm{B}}$ & $1.95 \times 10^{8 B}$ \\
\hline 3 & (o) & $6035.12^{\mathrm{A}}$ & 6265.21 & 2 & (e) & $64022.28^{\mathrm{A}}$ & 64010.70 & $1724.520^{\mathrm{A}}$ & $-2.772^{\mathrm{A}}$ & $3.80 \times 10^{6 A}$ \\
\hline & & & & & & & & $1721.974^{\mathrm{B}}$ & $-2.303^{\mathrm{B}}$ & $1.12 \times 10^{7 B}$ \\
\hline 0 & (e) & $32890.30^{\mathrm{B}}$ & 32838.62 & 1 & (o) & $93734.68^{\mathrm{A}}$ & 93602.83 & $1742.427^{\mathrm{A}}$ & $-2.432^{\mathrm{A}}$ & $8.13 \times 10^{6 A}$ \\
\hline & & & & & & & & $1746.956^{\mathrm{B}}$ & $-1.875^{\mathrm{B}}$ & $2.91 \times 10^{7 B}$ \\
\hline 2 & (o) & $7074.81^{\mathrm{B}}$ & 6571.36 & 1 & (e) & $63348.51^{\mathrm{A}}$ & 63335.40 & $1777.309^{\mathrm{A}}$ & $-2.207^{\mathrm{A}}$ & $1.31 \times 10^{7 A}$ \\
\hline & & & & & & & & $1772.294^{\mathrm{B}}$ & $-2.160^{\mathrm{B}}$ & $1.47 \times 10^{7 B}$ \\
\hline 2 & (o) & $9915.68^{\mathrm{B}}$ & 9900.49 & 3 & (e) & $65587.20^{\mathrm{A}}$ & 65550.73 & $1797.118^{\mathrm{A}}$ & $-1.882^{\mathrm{A}}$ & $2.71 \times 10^{7 A}$ \\
\hline & & & & & & & & $1800.738^{\mathrm{B}}$ & $-2.068^{\mathrm{B}}$ & $1.76 \times 10^{7 B}$ \\
\hline 1 & (o) & $8851.76^{\mathrm{A}}$ & 8922.05 & 2 & (e) & $64022.28^{\mathrm{A}}$ & 64010.70 & $1812.562^{\mathrm{A}}$ & $-1.804^{\mathrm{A}}$ & $3.19 \times 10^{7 A}$ \\
\hline & & & & & & & & $1810.333^{\mathrm{B}}$ & $-1.933^{\mathrm{B}}$ & $2.37 \times 10^{7 B}$ \\
\hline 1 & (o) & $8851.76^{\mathrm{A}}$ & 8922.05 & 1 & (e) & $63348.51^{\mathrm{A}}$ & 63335.40 & $1834.971^{\mathrm{A}}$ & $-1.469^{\mathrm{A}}$ & $6.72 \times 10^{7 A}$ \\
\hline & & & & & & & & $1829.441^{\mathrm{B}}$ & $-1.516^{\mathrm{B}}$ & $6.08 \times 10^{7 B}$ \\
\hline 3 & (o) & $10148.74^{\mathrm{A}}$ & 10126.53 & 2 & (e) & $64022.28^{\mathrm{A}}$ & 64010.70 & $1856.198^{\mathrm{A}}$ & $-1.756^{\mathrm{A}}$ & $3.39 \times 10^{7 A}$ \\
\hline & & & & & & & & $1851.373^{\mathrm{B}}$ & $-1.836^{\mathrm{B}}$ & $2.84 \times 10^{7 B}$ \\
\hline 2 & (o) & $9915.68^{\mathrm{B}}$ & 9900.49 & 1 & (e) & $63348.51^{\mathrm{A}}$ & 63335.40 & $1872.450^{\mathrm{A}}$ & $-1.979^{\mathrm{A}}$ & $2.00 \times 10^{7 A}$ \\
\hline & & & & & & & & $1866.259^{\mathrm{B}}$ & $-2.005^{\mathrm{B}}$ & $1.89 \times 10^{7 B}$ \\
\hline 3 & (o) & $12625.38^{\mathrm{B}}$ & 12500.72 & 3 & (e) & $65587.20^{\mathrm{A}}$ & 65550.73 & $1890.820^{\mathrm{A}}$ & $-1.623^{\mathrm{A}}$ & $4.44 \times 10^{7 A}$ \\
\hline & & & & & & & & $1893.111^{\mathrm{B}}$ & $-1.654^{\mathrm{B}}$ & $4.13 \times 10^{7 B}$ \\
\hline 1 & (o) & $18415.28^{\mathrm{A}}$ & 18443.63 & 2 & (e) & $70452.40^{\mathrm{B}}$ & 70433.08 & $1920.000^{\mathrm{A}}$ & $-0.896^{\mathrm{A}}$ & $2.30 \times 10^{8 A}$ \\
\hline & & & & & & & & $1918.052^{\mathrm{B}}$ & $-1.306^{\mathrm{B}}$ & $8.96 \times 10^{7 B}$ \\
\hline 0 & (o) & $11598.43^{\mathrm{A}}$ & 11577.16 & 1 & (e) & $63348.51^{\mathrm{A}}$ & 63335.40 & $1932.363^{\mathrm{A}}$ & $-1.595^{\mathrm{A}}$ & $4.53 \times 10^{7 A}$ \\
\hline & & & & & & & & $1927.433^{\mathrm{B}}$ & $-1.720^{\mathrm{B}}$ & $3.42 \times 10^{7 B}$ \\
\hline 2 & (o) & $12640.30^{\mathrm{B}}$ & 12641.55 & 2 & (e) & $64022.28^{\mathrm{A}}$ & 64010.70 & $1949.801^{\mathrm{A}}$ & $-1.750^{\mathrm{A}}$ & $3.12 \times 10^{7 A}$ \\
\hline & & & & & & & & $1944.183^{\mathrm{B}}$ & $-1.845^{\mathrm{B}}$ & $2.52 \times 10^{7 B}$ \\
\hline 2 & (o) & $19239.51^{\mathrm{A}}$ & 19236.23 & 2 & (e) & $70452.40^{\mathrm{B}}$ & 70433.08 & $1950.873^{\mathrm{A}}$ & $-2.865^{\mathrm{A}}$ & $2.39 \times 10^{6 A}$ \\
\hline & & & & & & & & $1950.102^{\mathrm{B}}$ & $-3.162^{\mathrm{B}}$ & $1.21 \times 10^{6 B}$ \\
\hline 3 & (o) & $19460.29^{\mathrm{A}}$ & 19464.46 & 2 & (e) & $70452.40^{\mathrm{B}}$ & 70433.08 & $1959.313^{\mathrm{A}}$ & $-0.667^{\mathrm{A}}$ & $3.74 \times 10^{8 A}$ \\
\hline & & & & & & & & $1958.901^{\mathrm{B}}$ & $-0.836^{\mathrm{B}}$ & $2.53 \times 10^{8 B}$ \\
\hline 2 & (o) & $12640.30^{\mathrm{B}}$ & 12641.55 & 1 & (e) & $63348.51^{\mathrm{A}}$ & 63335.40 & $1975.757^{\mathrm{A}}$ & $-2.774^{\mathrm{A}}$ & $2.88 \times 10^{6 A}$ \\
\hline & & & & & & & & $1966.237^{\mathrm{B}}$ & $-2.833^{\mathrm{B}}$ & $2.54 \times 10^{6 B}$ \\
\hline
\end{tabular}


TABLE II (cont.)

$\lambda, \log (g f)$, and $g A_{k i}$ for electric dipole (E1) transitions in Ce III. The lower and upper levels of each transition are presented by their $J$-values, parities [(e) for even and (o) for odd], and energy calculation results [ $\left.E_{\mathrm{th}}, 16\right]$ and experimental values [ $\left.E_{\mathrm{exp}}, 23\right]$.

\begin{tabular}{|c|c|c|c|c|c|c|c|c|c|c|}
\hline \multicolumn{8}{|c|}{ Transition } & \multirow{3}{*}{$\lambda[\AA]$} & \multirow{3}{*}{$\log (g f)$} & \multirow{3}{*}{$g A_{k i}\left[\mathrm{~s}^{-1}\right]$} \\
\hline \multicolumn{4}{|c|}{ Lower level } & \multicolumn{4}{|c|}{ Upper level } & & & \\
\hline$J$ & Parity & $E_{\mathrm{th}}\left[\mathrm{cm}^{-1}\right]$ & $E_{\exp }\left[\mathrm{cm}^{-1}\right]$ & $J$ & Parity & $E_{\mathrm{th}}\left[\mathrm{cm}^{-1}\right]$ & $E_{\exp }\left[\mathrm{cm}^{-1}\right]$ & & & \\
\hline \multirow[t]{2}{*}{4} & (o) & $5020.45^{\mathrm{A}}$ & 5127.27 & 5 & (e) & $53818.91^{\mathrm{A}}$ & 54193.84 & $2049.242^{\mathrm{A}}$ & $-2.157^{\mathrm{A}}$ & $1.11 \times 10^{7 A}$ \\
\hline & & & & & & & & $2053.944^{\mathrm{B}}$ & $-1.859^{\mathrm{B}}$ & $2.19 \times 10^{7 B}$ \\
\hline \multirow[t]{2}{*}{2} & (o) & $3843.05^{\mathrm{B}}$ & 3821.53 & 1 & (e) & $50769.20^{\mathrm{B}}$ & 51932.34 & $2220.756^{\mathrm{A}}$ & $-0.971^{\mathrm{A}}$ & $1.45 \times 10^{8 A}$ \\
\hline & & & & & & & & $2131.008^{\mathrm{B}}$ & $-0.858^{\mathrm{B}}$ & $2.04 \times 10^{8 B}$ \\
\hline \multirow[t]{2}{*}{5} & (o) & $6064.41^{\mathrm{A}}$ & 6361.27 & 5 & (e) & $53818.91^{\mathrm{A}}$ & 54193.84 & $2094.041^{\mathrm{A}}$ & $-1.017^{\mathrm{A}}$ & $1.46 \times 10^{8 A}$ \\
\hline & & & & & & & & $2097.356^{\mathrm{B}}$ & $-0.959^{\mathrm{B}}$ & $1.67 \times 10^{8 B}$ \\
\hline \multirow[t]{2}{*}{4} & (o) & $7262.13^{\mathrm{B}}$ & 7150.05 & 5 & (e) & $53818.91^{\mathrm{A}}$ & 54193.84 & $2148.629^{\mathrm{A}}$ & $-1.109^{\mathrm{A}}$ & $1.12 \times 10^{8 A}$ \\
\hline & & & & & & & & $2153.217^{\mathrm{B}}$ & $-0.936^{\mathrm{B}}$ & $1.67 \times 10^{8 B}$ \\
\hline \multirow[t]{2}{*}{4} & (o) & $7739.26^{\mathrm{B}}$ & 7836.72 & 5 & (e) & $53818.91^{\mathrm{A}}$ & 54193.84 & $2169.248^{\mathrm{A}}$ & $-2.123^{\mathrm{A}}$ & $1.07 \times 10^{7 A}$ \\
\hline & & & & & & & & $2175.567^{\mathrm{B}}$ & $-3.319^{\mathrm{B}}$ & $6.76 \times 10^{5 B}$ \\
\hline \multirow[t]{2}{*}{2} & (o) & $19239.51^{\mathrm{A}}$ & 19236.23 & 3 & (e) & $65587.20^{\mathrm{A}}$ & 65550.73 & $2157.604^{\mathrm{A}}$ & $-2.381^{\mathrm{A}}$ & $5.95 \times 10^{6 A}$ \\
\hline & & & & & & & & $2160.973^{\mathrm{B}}$ & $-2.365^{\mathrm{B}}$ & $6.17 \times 10^{6 B}$ \\
\hline \multirow[t]{2}{*}{2} & (o) & $3843.05^{\mathrm{B}}$ & 3821.53 & 2 & (e) & $49979.17^{\mathrm{B}}$ & 50043.85 & $2098.689^{\mathrm{A}}$ & $-3.303^{\mathrm{A}}$ & $7.53 \times 10^{5 A}$ \\
\hline & & & & & & & & $2167.500^{\mathrm{B}}$ & $-1.746^{\mathrm{B}}$ & $2.55 \times 10^{7 B}$ \\
\hline \multirow[t]{2}{*}{2} & (o) & $3843.05^{\mathrm{B}}$ & 3821.53 & 1 & (e) & $48639.61^{\mathrm{B}}$ & 48674.12 & $2157.082^{\mathrm{A}}$ & $-3.519^{\mathrm{A}}$ & $4.34 \times 10^{5 A}$ \\
\hline & & & & & & & & $2232.315^{\mathrm{B}}$ & $-3.375^{\mathrm{B}}$ & $5.64 \times 10^{5 B}$ \\
\hline \multirow[t]{2}{*}{2} & (o) & $19239.51^{\mathrm{A}}$ & 19236.23 & 2 & (e) & $64022.28^{\mathrm{A}}$ & 64010.70 & $2233.001^{\mathrm{A}}$ & $-0.851^{\mathrm{A}}$ & $1.88 \times 10^{8 A}$ \\
\hline & & & & & & & & $2227.037^{\mathrm{B}}$ & $-0.860^{\mathrm{B}}$ & $1.86 \times 10^{8 B}$ \\
\hline \multirow[t]{2}{*}{2} & (o) & $9915.68^{\mathrm{B}}$ & 9900.49 & 2 & (e) & $53775.10^{\mathrm{B}}$ & 54556.48 & $2217.268^{\mathrm{A}}$ & $-1.081^{\mathrm{A}}$ & $1.13 \times 10^{8 A}$ \\
\hline & & & & & & & & $2280.014^{\mathrm{B}}$ & $-1.818^{\mathrm{B}}$ & $1.95 \times 10^{7 B}$ \\
\hline 3 & (o) & $21851.41^{\mathrm{A}}$ & 21849.47 & 3 & (e) & $65587.20^{\mathrm{A}}$ & 65550.73 & $2286.458^{\mathrm{A}}$ & $-1.270^{\mathrm{A}}$ & $6.86 \times 10^{7 A}$ \\
\hline & & & & & & & & $2289.646^{\mathrm{B}}$ & $-1.283^{\mathrm{B}}$ & $6.63 \times 10^{7 B}$ \\
\hline 2 & (o) & $3843.05^{\mathrm{B}}$ & 3821.53 & 2 & (e) & $46835.73^{\mathrm{B}}$ & 46889.79 & $2358.312^{\mathrm{A}}$ & $-3.235^{\mathrm{A}}$ & $6.98 \times 10^{5 A}$ \\
\hline & & & & & & & & $2325.978^{\mathrm{B}}$ & $-3.359^{\mathrm{B}}$ & $5.39 \times 10^{5 B}$ \\
\hline 1 & (o) & $11615.86^{\mathrm{A}}$ & 11612.67 & 2 & (e) & $53775.10^{\mathrm{B}}$ & 54556.48 & $2302.702^{\mathrm{A}}$ & $-2.089^{\mathrm{A}}$ & $1.02 \times 10^{7 A}$ \\
\hline & & & & & & & & $2370.330^{\mathrm{B}}$ & $-1.743^{\mathrm{B}}$ & $2.15 \times 10^{7 B}$ \\
\hline 3 & (o) & $21851.41^{\mathrm{A}}$ & 21849.47 & 2 & (e) & $64022.28^{\mathrm{A}}$ & 64010.70 & $2371.306^{\mathrm{A}}$ & $-0.943^{\mathrm{A}}$ & $1.35 \times 10^{8 A}$ \\
\hline & & & & & & & & $2363.946^{\mathrm{B}}$ & $-0.931^{\mathrm{B}}$ & $1.40 \times 10^{8 B}$ \\
\hline 2 & (o) & $9915.68^{\mathrm{B}}$ & 9900.49 & 1 & (e) & $50769.20^{\mathrm{B}}$ & 51932.34 & $2561.638^{\mathrm{A}}$ & $-1.150^{\mathrm{A}}$ & $7.19 \times 10^{7 A}$ \\
\hline & & & & & & & & $2447.772^{\mathrm{B}}$ & $-1.137^{\mathrm{B}}$ & $8.12 \times 10^{7 B}$ \\
\hline 3 & (o) & $5479.13^{\mathrm{B}}$ & 5502.37 & 2 & (e) & $46835.73^{\mathrm{B}}$ & 46889.79 & $2450.137^{\mathrm{A}}$ & $-2.157^{\mathrm{A}}$ & $7.75 \times 10^{6 A}$ \\
\hline & & & & & & & & $2417.995^{\mathrm{B}}$ & $-2.102^{\mathrm{B}}$ & $9.01 \times 10^{6 B}$ \\
\hline 1 & (o) & $8851.76^{\mathrm{A}}$ & 8922.05 & 2 & (e) & $49979.17^{\mathrm{B}}$ & 50043.85 & $2339.323^{\mathrm{A}}$ & $-3.572^{\mathrm{A}}$ & $3.27 \times 10^{5 A}$ \\
\hline & & & & & & & & $2430.616^{\mathrm{B}}$ & $-2.173^{\mathrm{B}}$ & $7.57 \times 10^{6 B}$ \\
\hline 3 & (o) & $6035.12^{\mathrm{A}}$ & 6265.21 & 2 & (e) & $46835.73^{\mathrm{B}}$ & 46889.79 & $2480.252^{\mathrm{A}}$ & $-2.098^{\mathrm{A}}$ & $8.66 \times 10^{6 A}$ \\
\hline & & & & & & & & $2449.009^{\mathrm{B}}$ & $-2.111^{\mathrm{B}}$ & $8.62 \times 10^{6 B}$ \\
\hline 4 & (o) & $3372.91^{\mathrm{B}}$ & 3276.66 & 4 & (e) & $43767.92^{\mathrm{A}}$ & 43517.46 & $2479.155^{\mathrm{A}}$ & $-1.360^{\mathrm{A}}$ & $4.74 \times 10^{7 A}$ \\
\hline & & & & & & & & $2505.432^{\mathrm{B}}$ & $-1.509^{\mathrm{B}}$ & $3.29 \times 10^{7 B}$ \\
\hline 2 & (o) & $9915.68^{\mathrm{B}}$ & 9900.49 & 2 & (e) & $49979.17^{\mathrm{B}}$ & 50043.85 & $2400.580^{\mathrm{A}}$ & $-1.384^{\mathrm{A}}$ & $4.78 \times 10^{7 A}$ \\
\hline & & & & & & & & $2496.041^{\mathrm{B}}$ & $-2.137^{\mathrm{B}}$ & $7.81 \times 10^{6 B}$ \\
\hline 1 & (o) & $8851.76^{\mathrm{A}}$ & 8922.05 & 1 & (e) & $48639.61^{\mathrm{B}}$ & 48674.12 & $2412.106^{\mathrm{A}}$ & $-1.228^{\mathrm{A}}$ & $6.78 \times 10^{7 A}$ \\
\hline & & & & & & & & $2512.419^{\mathrm{B}}$ & $-1.287^{\mathrm{B}}$ & $5.45 \times 10^{7 B}$ \\
\hline 2 & (o) & $12640.30^{\mathrm{B}}$ & 12641.55 & 1 & (e) & $50769.20^{\mathrm{B}}$ & 51932.34 & $2758.996^{\mathrm{A}}$ & $-1.952^{\mathrm{A}}$ & $9.79 \times 10^{6 A}$ \\
\hline & & & & & & & & $2622.682^{\mathrm{B}}$ & $-1.914^{\mathrm{B}}$ & $1.18 \times 10^{7 B}$ \\
\hline 2 & (o) & $9915.68^{\mathrm{B}}$ & 9900.49 & 1 & (e) & $48639.61^{\mathrm{B}}$ & 48674.12 & $2477.286^{\mathrm{A}}$ & $-0.652^{\mathrm{A}}$ & $2.42 \times 10^{8 A}$ \\
\hline & & & & & & & & $2582.385^{\mathrm{B}}$ & $-0.677^{\mathrm{B}}$ & $2.11 \times 10^{8 B}$ \\
\hline 1 & (o) & $11615.86^{\mathrm{A}}$ & 11612.67 & 2 & (e) & $49979.17^{\mathrm{B}}$ & 50043.85 & $2501.044^{\mathrm{A}}$ & $-1.198^{\mathrm{A}}$ & $6.76 \times 10^{7 A}$ \\
\hline & & & & & & & & $2604.690^{\mathrm{B}}$ & $-0.983^{\mathrm{B}}$ & $1.02 \times 10^{8 B}$ \\
\hline 4 & (o) & $5020.45^{\mathrm{A}}$ & 5127.27 & 4 & (e) & $43767.92^{\mathrm{A}}$ & 43517.46 & $2580.811^{\mathrm{A}}$ & $-1.171^{\mathrm{A}}$ & $6.76 \times 10^{7 A}$ \\
\hline & & & & & & & & $2613.098^{\mathrm{B}}$ & $-1.224^{\mathrm{B}}$ & $5.84 \times 10^{7 B}$ \\
\hline 2 & (o) & $3843.05^{\mathrm{B}}$ & 3821.53 & 3 & (e) & $42008.62^{\mathrm{A}}$ & 41938.54 & $2627.552^{\mathrm{A}}$ & $-1.740^{\mathrm{A}}$ & $1.76 \times 10^{7 A}$ \\
\hline & & & & & & & & $2637.455^{\mathrm{B}}$ & $-1.832^{\mathrm{B}}$ & $1.41 \times 10^{7 B}$ \\
\hline
\end{tabular}


TABLE II (cont.)

$\lambda, \log (g f)$, and $g A_{k i}$ for electric dipole (E1) transitions in Ce III. The lower and upper levels of each transition are presented by their $J$-values, parities [(e) for even and (o) for odd], and energy calculation results [ $\left.E_{\mathrm{th}}, 16\right]$ and experimental values [ $\left.E_{\text {exp }}, 23\right]$.

\begin{tabular}{|c|c|c|c|c|c|c|c|c|c|c|}
\hline \multicolumn{8}{|c|}{ Transition } & \multirow{3}{*}{$\lambda[\AA]$} & \multirow{3}{*}{$\log (g f)$} & \multirow{3}{*}{$g A_{k i}\left[\mathrm{~s}^{-1}\right]$} \\
\hline \multicolumn{4}{|c|}{ Lower level } & \multicolumn{4}{|c|}{ Upper level } & & & \\
\hline$J$ & Parity & $E_{\mathrm{th}}\left[\mathrm{cm}^{-1}\right]$ & $E_{\exp }\left[\mathrm{cm}^{-1}\right]$ & $J$ & Parity & $E_{\mathrm{th}}\left[\mathrm{cm}^{-1}\right]$ & $E_{\exp }\left[\mathrm{cm}^{-1}\right]$ & & & \\
\hline \multirow[t]{2}{*}{5} & (o) & $16200.62^{\mathrm{B}}$ & 16152.32 & 5 & (e) & $53818.91^{\mathrm{A}}$ & 54193.84 & $2649.888^{\mathrm{A}}$ & $-2.126^{\mathrm{A}}$ & $7.11 \times 10^{6 A}$ \\
\hline & & & & & & & & $2666.406^{\mathrm{B}}$ & $-2.066^{\mathrm{B}}$ & $8.05 \times 10^{6 B}$ \\
\hline \multirow[t]{2}{*}{3} & (o) & $5479.13^{\mathrm{B}}$ & 5502.37 & 4 & (e) & $43767.92^{\mathrm{A}}$ & 43517.46 & $2615.857^{\mathrm{A}}$ & $-1.615^{\mathrm{A}}$ & $2.36 \times 10^{7 A}$ \\
\hline & & & & & & & & $2645.008^{\mathrm{B}}$ & $-1.751^{\mathrm{B}}$ & $1.69 \times 10^{7 B}$ \\
\hline \multirow[t]{2}{*}{3} & (o) & $12625.38^{\mathrm{B}}$ & 12500.72 & 2 & (e) & $49979.17^{\mathrm{B}}$ & 50043.85 & $2570.755^{\mathrm{A}}$ & $-0.653^{\mathrm{A}}$ & $2.24 \times 10^{8 A}$ \\
\hline & & & & & & & & $2677.106^{\mathrm{B}}$ & $-0.539^{\mathrm{B}}$ & $2.69 \times 10^{8 B}$ \\
\hline \multirow[t]{2}{*}{2} & (o) & $12640.30^{\mathrm{B}}$ & 12641.55 & 2 & (e) & $49979.17^{\mathrm{B}}$ & 50043.85 & $2573.065^{\mathrm{A}}$ & $-0.692^{\mathrm{A}}$ & $2.05 \times 10^{8 A}$ \\
\hline & & & & & & & & $2678.173^{\mathrm{B}}$ & $-0.986^{\mathrm{B}}$ & $9.60 \times 10^{7 B}$ \\
\hline \multirow[t]{2}{*}{3} & (o) & $6035.12^{\mathrm{A}}$ & 6265.21 & 4 & (e) & $43767.92^{\mathrm{A}}$ & 43517.46 & $2650.213^{\mathrm{A}}$ & $-2.449^{\mathrm{A}}$ & $3.37 \times 10^{6 A}$ \\
\hline & & & & & & & & $2682.165^{\mathrm{B}}$ & $-2.289^{\mathrm{B}}$ & $4.77 \times 10^{6 B}$ \\
\hline \multirow[t]{2}{*}{0} & (o) & $11598.43^{\mathrm{A}}$ & 11577.16 & 1 & (e) & $48639.61^{\mathrm{B}}$ & 48674.12 & $2583.252^{\mathrm{A}}$ & $-1.243^{\mathrm{A}}$ & $5.71 \times 10^{7 \mathrm{~A}}$ \\
\hline & & & & & & & & $2701.005^{\mathrm{B}}$ & $-1.333^{\mathrm{B}}$ & $4.25 \times 10^{7 B}$ \\
\hline \multirow[t]{2}{*}{1} & (o) & $11615.86^{\mathrm{A}}$ & 11612.67 & 1 & (e) & $48639.61^{\mathrm{B}}$ & 48674.12 & $2584.416^{\mathrm{A}}$ & $-1.265^{\mathrm{A}}$ & $5.42 \times 10^{7 A}$ \\
\hline & & & & & & & & $2698.856^{\mathrm{B}}$ & $-1.320^{\mathrm{B}}$ & $4.39 \times 10^{7 B}$ \\
\hline \multirow[t]{2}{*}{2} & (o) & $3843.05^{\mathrm{B}}$ & 3821.53 & 2 & (e) & $40305.48^{\mathrm{B}}$ & 40440.20 & $2756.523^{\mathrm{A}}$ & $-0.751^{\mathrm{A}}$ & $1.56 \times 10^{8 A}$ \\
\hline & & & & & & & & $2742.549^{\mathrm{B}}$ & $-0.834^{\mathrm{B}}$ & $1.30 \times 10^{8 B}$ \\
\hline \multirow[t]{2}{*}{1} & (o) & $11615.86^{\mathrm{A}}$ & 11612.67 & 0 & (e) & $48045.90^{\mathrm{B}}$ & 48075.96 & $2634.314^{\mathrm{A}}$ & $-1.375^{\mathrm{A}}$ & $4.05 \times 10^{7 A}$ \\
\hline & & & & & & & & $2742.806^{\mathrm{B}}$ & $-1.531^{\mathrm{B}}$ & $2.61 \times 10^{7 B}$ \\
\hline \multirow[t]{2}{*}{2} & (o) & $12640.30^{\mathrm{B}}$ & 12641.55 & 1 & (e) & $48639.61^{\mathrm{B}}$ & 48674.12 & $2661.393^{\mathrm{A}}$ & $-1.286^{\mathrm{A}}$ & $4.87 \times 10^{7 A}$ \\
\hline & & & & & & & & $2777.830^{\mathrm{B}}$ & $-1.451^{\mathrm{B}}$ & $3.06 \times 10^{7 B}$ \\
\hline \multirow[t]{2}{*}{2} & (o) & $7074.81^{\mathrm{B}}$ & 6571.36 & 3 & (e) & $42008.62^{\mathrm{A}}$ & 41938.54 & $2863.283^{\mathrm{A}}$ & $-2.057^{\mathrm{A}}$ & $7.14 \times 10^{6 A}$ \\
\hline & & & & & & & & $2883.206^{\mathrm{B}}$ & $-2.119^{\mathrm{B}}$ & $6.10 \times 10^{6 B}$ \\
\hline \multirow[t]{2}{*}{1} & (o) & $11615.86^{\mathrm{A}}$ & 11612.67 & 2 & (e) & $46835.73^{\mathrm{B}}$ & 46889.79 & $2878.713^{\mathrm{A}}$ & $-1.452^{\mathrm{A}}$ & $2.85 \times 10^{7 A}$ \\
\hline & & & & & & & & $2836.972^{\mathrm{B}}$ & $-1.465^{\mathrm{B}}$ & $2.84 \times 10^{7 B}$ \\
\hline \multirow[t]{2}{*}{3} & (o) & $5479.13^{\mathrm{B}}$ & 5502.37 & 2 & (e) & $40305.48^{\mathrm{B}}$ & 40440.20 & $2882.807^{\mathrm{A}}$ & $-1.053^{\mathrm{A}}$ & $7.11 \times 10^{7 A}$ \\
\hline & & & & & & & & $2871.390^{\mathrm{B}}$ & $-0.935^{\mathrm{B}}$ & $9.40 \times 10^{7 B}$ \\
\hline \multirow[t]{2}{*}{4} & (o) & $7262.13^{\mathrm{B}}$ & 7150.05 & 3 & (e) & $42008.62^{\mathrm{A}}$ & 41938.54 & $2879.275^{\mathrm{A}}$ & $-0.819^{\mathrm{A}}$ & $1.22 \times 10^{8 A}$ \\
\hline & & & & & & & & $2898.866^{\mathrm{B}}$ & $-0.724^{\mathrm{B}}$ & $1.50 \times 10^{8 B}$ \\
\hline 2 & (o) & $7074.81^{\mathrm{B}}$ & 6571.36 & 2 & (e) & $40305.48^{\mathrm{B}}$ & 40440.20 & $3017.111^{\mathrm{A}}$ & $-2.217^{\mathrm{A}}$ & $4.45 \times 10^{6 A}$ \\
\hline & & & & & & & & $3009.265^{\mathrm{B}}$ & $-2.019^{\mathrm{B}}$ & $7.04 \times 10^{6 B}$ \\
\hline 3 & (o) & $12625.38^{\mathrm{B}}$ & 12500.72 & 4 & (e) & $43767.92^{\mathrm{A}}$ & 43517.46 & $3218.762^{\mathrm{A}}$ & $-2.670^{\mathrm{A}}$ & $1.38 \times 10^{6 A}$ \\
\hline & & & & & & & & $3261.493^{\mathrm{B}}$ & $-2.615^{\mathrm{B}}$ & $1.52 \times 10^{6 B}$ \\
\hline 2 & (o) & $9915.68^{\mathrm{B}}$ & 9900.49 & 2 & (e) & $40305.48^{\mathrm{B}}$ & 40440.20 & $3301.920^{\mathrm{A}}$ & $-2.862^{\mathrm{A}}$ & $8.41 \times 10^{5 A}$ \\
\hline & & & & & & & & $3290.580^{\mathrm{B}}$ & $-2.975^{\mathrm{B}}$ & $6.53 \times 10^{5 B}$ \\
\hline 1 & (o) & $18415.28^{\mathrm{A}}$ & 18443.63 & 0 & (e) & $48045.90^{\mathrm{B}}$ & 48075.96 & $3209.124^{\mathrm{A}}$ & $-3.199^{\mathrm{A}}$ & $4.09 \times 10^{5 A}$ \\
\hline & & & & & & & & $3363.642^{\mathrm{B}}$ & $-2.833^{\mathrm{B}}$ & $8.66 \times 10^{5 B}$ \\
\hline 3 & (o) & $12625.38^{\mathrm{B}}$ & 12500.72 & 3 & (e) & $42008.62^{\mathrm{A}}$ & 41938.54 & $3411.976^{\mathrm{A}}$ & $-4.052^{\mathrm{A}}$ & $5.08 \times 10^{4 A}$ \\
\hline & & & & & & & & $3432.533^{\mathrm{B}}$ & $-3.901^{\mathrm{B}}$ & $7.12 \times 10^{4 B}$ \\
\hline 2 & (o) & $19239.51^{\mathrm{A}}$ & 19236.23 & 3 & (e) & $42008.62^{\mathrm{A}}$ & 41938.54 & $4391.912^{\mathrm{A}}$ & $-3.053^{\mathrm{A}}$ & $3.06 \times 10^{5 A}$ \\
\hline & & & & & & & & $4427.652^{\mathrm{B}}$ & $-3.015^{\mathrm{B}}$ & $3.28 \times 10^{5 B}$ \\
\hline 2 & (o) & $19239.51^{\mathrm{A}}$ & 19236.23 & 2 & (e) & $40305.48^{\mathrm{B}}$ & 40440.20 & $4764.520^{\mathrm{A}}$ & $-2.045^{\mathrm{A}}$ & $2.65 \times 10^{6 A}$ \\
\hline & & & & & & & & $4732.065^{\mathrm{B}}$ & $-1.919^{\mathrm{B}}$ & $3.59 \times 10^{6 B}$ \\
\hline 4 & (e) & $0.00^{\mathrm{B}}$ & 0.00 & 3 & (e) & $10148.74^{\mathrm{A}}$ & 10126.53 & $9853.452^{\mathrm{A}}$ & $-3.360^{\mathrm{A}}$ & $3.00 \times 10^{4 A}$ \\
\hline & & & & & & & & $9938.551^{\mathrm{B}}$ & $-3.209^{\mathrm{B}}$ & $4.17 \times 10^{4 B}$ \\
\hline
\end{tabular}

